\title{
Generalized reduced magnetohydrodynamic equations
}

\author{
S. E. Kruger, ${ }^{\text {a) }}$ C. C. Hegna, and J. D. Callen \\ Department of Engineering Physics, 1500 Engineering Drive, Madison, Wisconsin 53706-1687
}

(Received 28 July 1998; accepted 24 August 1998)

\begin{abstract}
A new derivation of reduced magnetohydrodynamic (MHD) equations is presented. A multiple-time-scale expansion is employed. It has the advantage of clearly separating the three time scales of the problem associated with (1) MHD equilibrium, (2) fluctuations whose wave vector is aligned perpendicular to the magnetic field, and (3) those aligned parallel to the magnetic field. The derivation is carried out without relying on a large aspect ratio assumption; therefore this model can be applied to any general toroidal configuration. By accounting for the MHD equilibrium and constraints to eliminate the fast perpendicular waves, equations are derived to evolve scalar potential quantities on a time scale associated with the parallel wave vector (shear-Alfven wave time scale), which is the time scale of interest for MHD instability studies. Careful attention is given in the derivation to satisfy energy conservation and to have manifestly divergence-free magnetic fields to all orders in the expansion parameter. Additionally, neoclassical closures and equilibrium shear flow effects are easily accounted for in this model. Equations for the inner resistive layer are derived which reproduce the linear ideal and resistive stability criterion of Glasser, Greene, and Johnson [Phys. Fluids 18, 875 (1975)]. C 1998 American Institute of Physics. [S1070-664X(98)02012-6]
\end{abstract}

\section{INTRODUCTION}

Reduced descriptions of the magnetohydrodynamic (MHD) equations have a number of attractive features for theoretical and numerical calculations. ${ }^{1-7}$ The goal of these descriptions is a reduced set of equations that embody the most salient physics of MHD stability properties in magnetized, toroidal plasmas. These reduced models eliminate the fast time-scale magnetosonic waves, which significantly constrain the computational speed of solving the full MHD equations and do not significantly contribute to instabilities. Strauss ${ }^{1}$ introduced these models by reducing the MHD equations using the inverse aspect ratio of the torus as the expansion parameter. Hazeltine and Meiss ${ }^{5}$ furthered the basic physics understanding of reduced MHD by giving a derivation using $k_{\|} / k_{\perp}$ as the expansion parameter, which was introduced as a means to eliminate the fast time scale associated with motions perpendicular to the magnetic field. The Hazeltine and Meiss derivation is a superset of the original derivation in Ref. 1 since in large aspect ratio devices, this expansion parameter scales as

$$
\frac{k_{\|}}{k_{\perp}} \sim \frac{a}{R q} \ll 1,
$$

where $a, R$, and $q$ are the plasma minor radius, major radius, and safety factor, respectively, which is formally small in the large aspect ratio tokamak ordering.

Despite the success in heuristically explaining the fundamental physics of reduced MHD equations, some aspects of the derivation given by Hazeltine and Meiss are unsatisfactory. A separation between the equilibrium length scale and parallel length scale is not made, which makes the identification of the MHD equilibrium conditions difficult. More

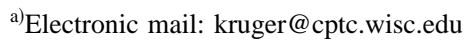

seriously, however, their form of the perturbed magnetic field is not divergence free and energy is not conserved to all orders. The goal of the present work is to derive a set of reduced equations which do not have these inadequacies and to further clarify the physics of reduced MHD.

A recent derivation of reduced equations by Strauss ${ }^{6}$ has many similarities to the derivation by Hazeltine and Meiss and the derivation in this work. In Ref. 6, an expansion is made around a large "vacuum" magnetic field that is divergence and curl-free. The expansion parameter used is the ratio of the induced field to the vacuum magnetic field which can be shown to be consistent with $k_{\|} / k_{\perp}$ being small. Contrary to what is stated in the paper, however, this derivation is not valid for low-aspect ratio tokamaks because such devices typically have induced magnetic fields comparable to the vacuum magnetic field. ${ }^{8,9}$

We also note that the same length-scale ordering used here has recently been applied to the ideal MHD Lagrangian for studying ballooning modes in three-dimensional configurations. ${ }^{10}$ Although the motivations are different, there are many parallels to this work and it provides a useful contrast to this paper.

In this paper, a set of reduced MHD equations, which are valid at any aspect ratio, are derived using $k_{\|} / k_{\perp}$ as a small expansion parameter. In Sec. II, the ordering is introduced and shown to naturally lead to a multiple time- and lengthscale expansion. Then it is applied to the MHD equations. The fundamental assumptions of reduced MHD are elucidated by examining the behavior on the fastest time scale. In Sec. III, equations having the same structure as other reduced equations are derived that evolve only scalar quantities and satisfy the constraints required to eliminate the fast motions. Energy conservation and the procedure for maintaining a divergence-free magnetic field are shown. An additional advantage to the model introduced here relative to previous 
reduced MHD models is the ability to introduce additional physics. In this section, we also discuss two additional physics effects: the self-consistent incorporation of sub-Alfvenic equilibrium flow profiles and neoclassical closures. In Sec. IV, we develop a linear stability analysis for resistive modes using the reduced equations derived in this paper based on a thin-layer width approximation. Finally, in Sec. V we discuss and summarize the findings.

\section{FUNDAMENTALS OF REDUCED MHD}

The ordering used in this derivation is designed to look at modes whose wavelengths are small compared to the minor radius a (equilibrium scale length) and to the parallel wavelength, i.e.,

$$
\frac{\lambda_{\perp}}{\lambda_{\|}} \sim \epsilon \quad \text { and } \frac{\lambda_{\perp}}{a} \sim \epsilon,
$$

where $\epsilon \ll 1$, and the wavelength ordering is defined for directions relative to a large-scale magnetic field. Since magnetic shear localizes modes in the perpendicular direction, many MHD instabilities are characterized by this ordering. These perturbations are called "flute",5,10 perturbations because the magnetic surfaces of such perturbations resemble a fluted column.

To formally obtain the ordering of Eq. (2), we order the space and time dependencies of first-order quantities as

$$
Q_{1}=Q_{1}\left(\frac{\vec{x}_{\perp}}{\epsilon}, \vec{x}_{\|}, \frac{t_{\perp}}{\epsilon}, t_{\|}\right),
$$

where the notation $\vec{x}_{\perp}\left(\vec{x}_{\|}\right)$denotes spatial dependence of perturbed quantities in the direction perpendicular (parallel) to the zeroth-order magnetic field, and $t_{\perp}$ and $t_{\|}$are the time scales associated with their respective spatial scales. When applying the gradient operator to a perturbed quantity $Q_{1}$, this ordering of the spatial scales gives the desired small $k_{\|} / k_{\perp}$ :

$$
\vec{\nabla} Q_{1}=\left(\frac{1}{\epsilon} \vec{\nabla}_{\perp}+\vec{\nabla}_{\|}\right) Q_{1}\left(\vec{x}_{\perp}, \vec{x}_{\|}\right) .
$$

The time scales have the same ordering as the spatial scales:

$$
\frac{\partial Q_{1}}{\partial t}=\left(\frac{1}{\epsilon} \frac{\partial}{\partial t_{\perp}}+\frac{\partial}{\partial t_{\|}}\right) Q_{1} .
$$

The terms parallel and perpendicular here are defined with respect to a zeroth-order magnetic field such that

$$
\vec{\nabla}_{\|} \equiv \hat{\mathbf{b}}_{\mathbf{0}}\left(\hat{\mathbf{b}}_{\mathbf{0}} \cdot \vec{\nabla}\right) ; \quad \vec{B}_{0} \cdot \vec{\nabla}_{\perp} Q_{1}=0
$$

where $\hat{\mathbf{b}}_{0} \equiv \vec{B}_{0} / B_{0}$. Because the expansion is performed in terms of anisotropic, spatially dependent operators, which introduces subtleties in the derivation, further properties of the perpendicular and parallel gradient operators are given in Appendix A. The expansion parameter is given by the anisotropy of the perturbed response; therefore the zeroth-order quantities are ordered as

$$
Q_{0}=Q_{0}(\vec{x}, t)
$$

so that their spatial scales $(\vec{x})$ and time scales $(\mathrm{t})$ are not separated by direction.

The MHD variables are ordered then as

$$
\begin{aligned}
& \rho=\rho_{0}(\vec{x}, t)+\epsilon \rho_{1}\left(\frac{\vec{x}_{\perp}}{\epsilon}, \vec{x}_{\|}, \frac{t_{\perp}}{\epsilon}, t_{\|}\right) ; \\
& p=p_{0}(\vec{x}, t)+\epsilon p_{1}\left(\frac{\vec{x}_{\perp}}{\epsilon}, \vec{x}_{\|}, \frac{t_{\perp}}{\epsilon}, t_{\|},\right) ; \\
& \vec{V}=\epsilon \vec{V}_{1}\left(\frac{\vec{x}_{\perp}}{\epsilon}, \vec{x}_{\|}, \frac{t_{\perp}}{\epsilon}, t_{\|}\right) ; \\
& \vec{B}=\vec{B}_{0}(\vec{x}, t)+\epsilon \vec{B}_{1}\left(\frac{\vec{x}_{\perp}}{\epsilon}, \vec{x}_{\|}, \frac{t_{\perp}}{\epsilon}, t_{\|}\right), \\
& \Pi=\epsilon \Pi_{0}(\vec{x}, t)+\epsilon \Pi_{1}\left(\frac{\vec{x}_{\perp}}{\epsilon}, \vec{x}_{\|}, \frac{t_{\perp}}{\epsilon}, t_{\|}\right) ; \\
& \Pi_{e}=\epsilon^{2} \Pi_{0}(\vec{x}, t)+\epsilon^{2} \Pi_{1}\left(\frac{\vec{x}_{\perp}}{\epsilon}, \vec{x}_{\|}, \frac{t_{\perp}}{\epsilon}, t_{\|}\right),
\end{aligned}
$$

where $\rho, p, \vec{B}, \vec{V}, \Pi$, and $\Pi_{e}$ are the plasma density, pressure, magnetic field, flow velocity field, total stress tensor, and electron stress tensor, respectively. Note that no assumptions are made on the zeroth-order quantities a priori (i.e., it is not assumed that they satisfy the usual MHD equilibrium force balance). This derivation most significantly differs from previous derivations of reduced equations by explicitly keeping the zeroth-order and the perpendicular time scales. They are kept here because the multiple-time-scale analysis allows one to observe the behavior of motions on these time scales as well as the desired $t_{\|}$time scale. The stress tensor $\Pi$ is the sum of both the ion and electron contributions and is ordered $\epsilon$. The electron stress tensor $\Pi_{e}$ and the resistivity, $\eta$, are ordered as $\epsilon^{2}$.

We apply the ordering of Eq. (8) to the MHD equations including the anisotropic stress tensor, but neglecting heat flows:

$$
\frac{\partial \rho}{\partial t}+(\vec{V} \cdot \vec{\nabla}) \rho+\rho(\nabla \cdot \vec{V})=0
$$

$$
\begin{aligned}
\frac{\partial p}{\partial t}+ & (\vec{V} \cdot \vec{\nabla}) p+\Gamma p(\nabla \cdot \vec{V}) \\
& =(\Gamma-1)\left[\eta J^{2}-\Pi: \vec{\nabla} \vec{V}-\frac{1}{n e} \Pi_{e}: \vec{\nabla} \vec{V}_{e}\right],
\end{aligned}
$$

$$
\begin{aligned}
& \frac{\partial \vec{B}}{\partial t}+(\vec{V} \cdot \vec{\nabla}) \vec{B}+\vec{B}(\nabla \cdot \vec{V})-(\vec{B} \cdot \vec{\nabla}) \vec{V} \\
& \quad=-\vec{\nabla} \times \eta \vec{\nabla} \times \vec{B}-\vec{\nabla} \times \frac{1}{n e} \vec{\nabla} \cdot \Pi_{e}, \\
& \rho\left(\frac{\partial \vec{V}}{\partial t}+(\vec{V} \cdot \vec{\nabla}) \vec{V}\right)=-\vec{\nabla}\left(p+B^{2} / 2\right)+(\vec{B} \cdot \vec{\nabla}) \vec{B}-\vec{\nabla} \cdot \Pi,
\end{aligned}
$$

in which we have set $\mu_{0}=1$. The $\mathrm{O}(1)$ equations are 


$$
\begin{aligned}
& -\frac{\partial \rho_{0}}{\partial t}=\frac{\partial \rho_{1}}{\partial t_{\perp}}+\rho_{0} \vec{\nabla}_{\perp} \cdot \vec{V}_{1}, \\
& -\frac{\partial p_{0}}{\partial t}=\frac{\partial p_{1}}{\partial t_{\perp}}+\Gamma p_{0} \vec{\nabla}_{\perp} \cdot \vec{V}_{1}, \\
& -\frac{\partial \vec{B}_{0}}{\partial t}=\frac{\partial \vec{B}_{1}}{\partial t_{\perp}}+\vec{B}_{0} \vec{\nabla}_{\perp} \cdot \vec{V}_{1}, \\
& -\vec{\nabla}\left(p_{0}+B_{0}^{2} / 2\right)+\left(\vec{B}_{0} \cdot \vec{\nabla}\right) \vec{B}_{0}=\rho_{0} \frac{\partial \vec{V}_{1}}{\partial t_{\perp}}+\vec{\nabla}_{\perp}\left(p_{1}+\vec{B}_{0} \cdot \vec{B}_{1}\right) .
\end{aligned}
$$

We assume that the zeroth-order quantities do not vary on the perpendicular time scale and assume wavelike solutions for the first-order quantities:

$$
\rho_{1}, p_{1}, \vec{V}_{1}, \vec{B}_{1} \sim e^{i\left(\vec{k}_{\perp} \cdot \vec{x}_{\perp}-\omega t_{\perp}\right)} .
$$

A perpendicular time-scale average is introduced and given by

$$
\langle Q\rangle_{t_{\perp}} \equiv \frac{1}{T_{\perp}} \int_{0}^{T_{\perp}} Q d t_{\perp} .
$$

This averaging operator is applied to Eqs. (10) to yield the longer time-scale behavior of these leading-order equations. After taking this average, the terms on the right side vanish and leave

$$
\begin{aligned}
& \left\langle\frac{\partial \rho_{0}}{\partial t}\right\rangle_{t_{\perp}}=\frac{\partial \rho_{0}}{\partial t}=0, \\
& \left\langle\frac{\partial p_{0}}{\partial t}\right\rangle_{t_{\perp}}=\frac{\partial p_{0}}{\partial t}=0, \\
& \left\langle\frac{\partial \vec{B}_{0}}{\partial t}\right\rangle_{t_{\perp}}=\frac{\partial \vec{B}_{0}}{\partial t}=0, \\
& \left\langle-\vec{\nabla}\left(p_{0}+B_{0}^{2} / 2\right)+\left(\vec{B}_{0} \cdot \vec{\nabla}\right) \vec{B}_{0}\right\rangle_{t_{\perp}} \\
& =-\vec{\nabla}\left(p_{0}+B_{0}^{2} / 2\right)+\left(\vec{B}_{0} \cdot \vec{\nabla}\right) \vec{B}_{0}=0,
\end{aligned}
$$

which shows that the zeroth-order quantities satisfy the MHD equilibrium equations exactly.

The equilibrium equation shows the need for a separation between the equilibrium length scale $(\vec{x})$ and the parallel length scale $\left(\vec{x}_{\|}\right)$, which was not done in the Hazeltine and Meiss derivation which used $Q_{0}=Q_{0}\left(\vec{x}_{\|}\right)$. The nontrivial projection of the last equation is in the perpendicular direction: $\vec{\nabla}_{\perp}\left(p_{0}+B_{0}^{2} / 2\right)=B_{0}^{2} \vec{\kappa}$, where $\vec{\kappa} \equiv\left(\hat{\mathbf{b}}_{0} \cdot \vec{\nabla}\right) \hat{\mathbf{b}}_{0}$ is the curvature vector associated with the lowest order magnetic field. The formulation of Hazeltine and Meiss would give $\vec{\nabla}_{\perp} p_{0}$ $=0$. Conceptually, such a separation of equilibrium and parallel length scales is desirable because the expansion parameter is then based on the anisotropy of the perturbation response, rather than the scale lengths of the equilibrium.

Equation (13) is used to eliminate zeroth-order quantities. Then the perturbed parts of Eqs. (10) can be written as

$$
\begin{aligned}
& \left(\frac{\partial^{2}}{\partial t_{\perp}^{2}}-\left(V_{S}^{2}+V_{A}^{2}\right) \vec{\nabla}_{\perp}^{2}\right)\left(p_{1}+\vec{B}_{0} \cdot \vec{B}_{1}\right)=0, \\
& \frac{\partial^{2} \vec{V}_{1}}{\partial t_{\perp}^{2}}-\vec{\nabla}_{\perp}\left(V_{S}^{2}+V_{A}^{2}\right) \vec{\nabla}_{\perp} \cdot \vec{V}_{1}=0 \\
& \frac{\partial^{2} \rho_{1}}{\partial t_{\perp}^{2}}-\vec{\nabla}_{\perp}^{2}\left(p_{1}+\vec{B}_{0} \cdot \vec{B}_{1}\right)=0 \\
& \frac{\partial^{2} \vec{B}_{1}}{\partial t_{\perp}^{2}}-\frac{\vec{B}_{0}}{\rho_{0}} \vec{\nabla}_{\perp}^{2}\left(p_{1}+\vec{B}_{0} \cdot \vec{B}_{1}\right)=0
\end{aligned}
$$

where $V_{S}^{2}=\Gamma p_{0} / \rho_{0}$ and $V_{A}^{2}=\vec{B}_{0}^{2} / \rho_{0}$. These are the leadingorder equations for fast magnetosonic waves that justifies the wavelike behavior assumed. Magnetosonic wave motions do not significantly contribute to linear MHD stability, ${ }^{11}$ but place a severe Courant-Friedrichs-Lewy (CFL) numerical constraint $^{12}$ on numerical solutions of the full MHD equations. To eliminate the fast, perpendicular time scale in the equations (i.e., to obtain $\partial Q_{1} / \partial t_{\perp}=0$ ), one chooses

$$
\vec{\nabla}_{\perp} \cdot \vec{V}_{1}=O(\epsilon), \quad p_{1}+\vec{B}_{0} \cdot \vec{B}_{1}=O(\epsilon)
$$

as constraints on our equations. These constraints form the basis of the reduction of the MHD equations, and explicitly show the reduced MHD assumption corresponds to fast magnetosonic waves equilibrating to the ideal MHD equilibrium, Eqs. (13).

With these constraints, the $O(\epsilon)$ equations become

$$
\begin{aligned}
& -\left(\frac{\partial \rho_{2}}{\partial t_{\perp}}+\rho_{0}\left(\vec{\nabla}_{\perp} \cdot \vec{V}_{2}\right)\right) \\
& =\frac{\partial \rho_{1}}{\partial t_{\|}}+\left(\vec{V}_{1} \cdot \vec{\nabla}_{\perp}\right) \rho_{1}+\left(\vec{V}_{1} \cdot \vec{\nabla}\right) \rho_{0}+\rho_{0}\left(\vec{\nabla}_{\|} \cdot \vec{V}_{1}\right), \\
& -\left(\frac{\partial p_{2}}{\partial t_{\perp}}+\Gamma p_{0}\left(\vec{\nabla}_{\perp} \cdot \vec{V}_{2}\right)\right) \\
& =\frac{\partial p_{1}}{\partial t_{\|}}+\left(\vec{V}_{1} \cdot \vec{\nabla}_{\perp}\right) p_{1}+\left(\vec{V}_{1} \cdot \vec{\nabla}\right) p_{0}+\Gamma p_{0}\left(\vec{\nabla}_{\|} \cdot \vec{V}_{1}\right) \\
& -(\Gamma-1)\left[\eta J^{2}-\Pi: \vec{\nabla} \vec{V}_{1}-\frac{1}{n e} \Pi_{e}: \vec{\nabla} \vec{V}_{e}\right], \\
& -\left(\frac{\partial \vec{B}_{2}}{\partial t_{\perp}}+\vec{B}_{0}\left(\vec{\nabla}_{\perp} \cdot \vec{V}_{2}\right)\right) \\
& =\frac{\partial \vec{B}_{1}}{\partial t_{\|}}+\left(\vec{V} \cdot \vec{\nabla}_{\perp}\right) \vec{B}_{1}-\left(\vec{B}_{1} \cdot \vec{\nabla}_{\perp}\right) \vec{V}_{1}-\left(\vec{B}_{1} \cdot \vec{\nabla}\right) \vec{V}_{0} \\
& +\eta \vec{\nabla}^{2} B_{1}+\vec{\nabla} \times \frac{1}{n e} \vec{\nabla} \cdot \Pi_{e}, \\
& -\left(\rho_{0} \frac{\partial \vec{V}_{2}}{\partial t_{\perp}}+\vec{\nabla}_{\perp}\left(p_{2}+\vec{B}_{0} \cdot \vec{B}_{2}\right)\right) \\
& =\rho_{0}\left(\frac{\partial \vec{V}_{1}}{\partial t_{\|}}+\left(\vec{V}_{1} \cdot \vec{\nabla}_{\perp}\right) \vec{V}_{1}\right)+\vec{\nabla}_{\perp} \frac{B_{1}^{2}}{2}+\vec{\nabla}_{\|}\left(\vec{B}_{0} \cdot \vec{B}_{1}\right)+\vec{\nabla}_{\|} p_{1} \\
& -\left(\vec{B}_{0} \cdot \vec{\nabla}_{\|}\right) \vec{B}_{1}-\left(\vec{B}_{1} \cdot \vec{\nabla}_{\perp}\right) \vec{B}_{1}-\left(\vec{B}_{1} \cdot \vec{\nabla}\right) \vec{B}_{0}+\vec{\nabla} \cdot \Pi .
\end{aligned}
$$


As before, the left side of these equations describes fast magnetosonic waves for the second-order quantities. These motions can again be eliminated using the averaging operator in Eq. (12), and one is left with averages of the right side over the fast $\left(t_{\perp}\right)$ time scale. As discussed later, parts of $\vec{V}_{2}$ will be kept in the derivation which cause $\vec{\nabla}_{\perp} \cdot \vec{V}_{2} \neq 0$ so that energy conservation is guaranteed; however, $\partial Q_{2} / \partial t_{\perp}=0$ will be assumed.

The remaining equations have eight variables. Equations (15) and $\vec{\nabla} \cdot \vec{B}=0$ introduce three constraints which leaves five fundamental variables. In the following section, Eqs. (16) are used to derive five equations to evolve five scalar quantities.

\section{REDUCED MHD EQUATIONS}

\section{A. Derivation}

In this section evolution equations for five scalar variables are derived using the information deduced from the lowest order equations of the previous section:

(1) If the constraints of Eq. (15) are satisfied, quantities do not vary on the perpendicular time scale.

(2) Zeroth-order quantities satisfy an equilibrium equation and do not vary with time.

Before beginning the derivation, energy conservation is briefly considered. Energy conservation for the MHD equations is shown by casting the equations in the form ${ }^{13}$

$$
\frac{\partial w}{\partial t}+\vec{\nabla} \cdot \vec{s}=0
$$

where $w$ is the energy density and $\vec{s}$ is the energy flux. Such an equation is integrated over all space to show that $(\partial / \partial t) \int w d V=0$ in the absence of energy flow through the boundary. Terms like $\vec{B}_{1} \cdot \vec{\nabla}_{\perp}$ in our $O(\epsilon)$ equations will cause the energy conservation equation to be in the form

$$
\frac{\partial w}{\partial t}+\vec{\nabla} \cdot \overrightarrow{s_{1}}+\vec{\nabla}+\overrightarrow{s_{2}}=0
$$

When integrated over all space, this equation will not satisfy energy conservation to all orders in $\epsilon$ because the $\vec{\nabla}_{\perp}$ operator is spatially dependent and terms containing it will not vanish. To correct this, we need to include $\vec{\nabla}_{\|}$terms that are formally lower in the ordering (such as $\vec{B}_{1} \cdot \vec{\nabla}_{\|}$) to produce complete divergences. However, for simplicity the lowerorder terms which are kept will not be shown explicitly. Keeping lower-order terms to satisfy energy conservation in this work is similar to the derivation of reduced equations given by Drake and Antonsen. ${ }^{3}$

Beginning with the right side of Eq. (16) and keeping the lower-order terms as mentioned, the pressure equation becomes

$$
\begin{aligned}
& \frac{d p_{1}}{d t_{\|}}+\left(\vec{V}_{1} \cdot \vec{\nabla}\right) p_{0}+\Gamma p_{T}\left(\nabla \cdot \vec{V}_{1}\right) \\
& \quad=(\Gamma-1)\left[\eta\left(J_{T_{\|}}^{2}+\frac{\left|\vec{\nabla} p_{T}\right|^{2}}{B_{0}^{2}}\right)-\Pi: \vec{\nabla} \vec{V}_{1}+\frac{1}{n e} \Pi_{e}: \vec{\nabla} \frac{\vec{J}}{n e}\right],
\end{aligned}
$$

which can be written in a form useful for showing energy conservation as

$$
\begin{aligned}
& \frac{\partial}{\partial t_{\|}} \frac{p_{1}}{\Gamma-1}+\vec{\nabla} \cdot \frac{\Gamma p_{T}}{\Gamma-1} \vec{V}_{1}-\left(\vec{V}_{1} \cdot \vec{\nabla} p_{T}\right) \\
& \quad=\eta\left(J_{T_{\|}}^{2}+\frac{\left|\vec{\nabla} p_{T}\right|^{2}}{B_{0}^{2}}\right)-\Pi: \vec{\nabla} \vec{V}+\frac{1}{n e} \Pi_{e}: \vec{\nabla} \frac{\vec{J}}{n e},
\end{aligned}
$$

where

$$
\begin{aligned}
& \frac{d}{d t_{\|}}=\frac{\partial}{\partial t_{\|}}+\left(\vec{V}_{1} \cdot \vec{\nabla}\right), \\
& \hat{\mathbf{b}}_{T} \equiv \hat{\mathbf{b}}_{0}+\hat{\mathbf{b}}_{1}=\frac{\vec{B}_{0}}{B_{0}}+\frac{\vec{B}_{1}}{B_{0}}=\frac{\vec{B}_{T}}{B_{0}}, \\
& \vec{B}_{T} \equiv \vec{B}_{0}+\vec{B}_{1}, \quad p_{T}=p_{0}+p_{1}, \\
& J_{T_{\|}}=J_{\| 0}+\widetilde{J}_{\|}=\hat{\mathbf{b}}_{0} \cdot \vec{\nabla} \times \vec{B}_{0}+\hat{\mathbf{b}}_{0} \cdot \vec{\nabla}_{\perp} \times \vec{B}_{1} .
\end{aligned}
$$

Here, the term $\vec{\nabla} \cdot \vec{V}_{1}$ is $\vec{\nabla}_{\|} \cdot \vec{V}_{1}+\vec{\nabla}_{\perp} \cdot \vec{V}_{2}$ and the perturbed parallel current has been denoted with a tilde rather than a subscript 1 because it is of order unity. The density equation when ordered is similar to Eq. (19) in form:

$$
\frac{d \rho_{1}}{d t_{\|}}+\rho_{T} \nabla \cdot \vec{V}_{1}=0
$$

where $\rho_{T} \equiv \rho_{0}+\rho_{1}$. Taking the parallel component of the momentum equation given in Eq. (16) gives

$$
\rho_{T} \frac{d V_{\|}}{d t_{\|}}=-\hat{\mathbf{b}}_{0} \cdot \vec{\nabla} p_{1}-\hat{\mathbf{b}}_{1} \cdot \vec{\nabla} p_{T}-\hat{\mathbf{b}}_{0} \cdot \vec{\nabla} \cdot \Pi,
$$

where $V_{\|} \equiv \vec{V}_{1} \cdot \hat{\mathbf{b}}_{0}$.

To derive equations for the perpendicular components of the magnetic induction equation and the momentum equation of Eqs. (16), it is easier to recast the ordering process in terms of the the electrostatic and magnetic potentials. These are ordered as

$$
\Phi=\epsilon^{2} \phi, \quad \vec{A}=\vec{A}_{0}+\epsilon^{2} \vec{A}_{2},
$$

such that the electric and magnetic fields are

$$
\begin{aligned}
& \vec{E}=-\vec{\nabla} \Phi-\frac{\partial \vec{A}}{\partial t}=\epsilon\left(-\vec{\nabla}_{\perp} \phi\right)+\epsilon^{2}\left(-\vec{\nabla}_{\|} \phi-\frac{\partial \vec{A}_{2}}{\partial t_{\|}}+\vec{E}^{A}\right), \\
& \vec{B}=\vec{\nabla} \times \vec{A}=\vec{\nabla} \times \vec{A}_{0}+\epsilon \vec{\nabla}_{\perp} \times \vec{A}_{2},
\end{aligned}
$$

where $\vec{E}^{A}$ is the applied electric field which is ordered to be consistent with the transport time-scale Ohm's law. Since we have eliminated motions on the perpendicular time scale, $\vec{E}$ is electrostatic to first order (i.e., $\partial \vec{A}_{2} / \partial t_{\perp}=0$ ). 
We now look at Ohm's Law and recall that the resistivity is ordered as $\eta \sim \epsilon^{2}$. The first order Ohm's Law is $\vec{E}_{1}$ $+\vec{V}_{1} \times \vec{B}_{0}=0$, which allows us to write

$$
\vec{V}_{1}=\frac{\vec{B}_{0} \times \vec{\nabla} \phi}{B_{0}^{2}}+V_{\|} \hat{\mathbf{b}}_{0} .
$$

The perpendicular divergence is (see Appendix

$$
\begin{aligned}
\vec{\nabla}_{\perp} \cdot \vec{V}_{1}= & \underbrace{\hat{\mathbf{b}}_{0} \cdot \vec{\nabla}_{\perp} V_{\|}+\frac{\vec{B}_{0}}{B_{0}^{2}} \cdot \vec{\nabla}_{\perp} \times \vec{\nabla}_{\perp} \phi}_{=0} \\
& +\underbrace{V_{\|} \vec{\nabla}_{\perp} \cdot \hat{\mathbf{b}}_{0}+\phi \vec{\nabla}_{\perp} \times \frac{\vec{B}_{0}}{B_{0}^{2}}}_{\sim \epsilon}=O(\epsilon),
\end{aligned}
$$

which satisfies the needed constraint in Eq. (15) to the desired order.

Looking at the Ohm's Law in the next order to get an expression for $\vec{V}_{2}$, we find

$$
\begin{aligned}
\vec{V}_{2_{\perp}}= & \frac{-B_{\| 1} \hat{\mathbf{b}}_{0} \times \vec{\nabla} \phi}{B_{0}^{2}}+V_{\|} \hat{\mathbf{b}}_{1}-\eta \frac{\vec{\nabla} p_{T}}{B_{0}^{2}} \\
& -\frac{\vec{B}_{0} \times(1 / n e) \vec{\nabla} \cdot \Pi_{e}}{B_{0}^{2}}+\frac{\partial}{\partial t_{\|}} \frac{\vec{B}_{0} \times \vec{A}_{2}}{B_{0}^{2}}-\frac{B_{\| 1}}{B_{0}} V_{\|} \hat{\mathbf{b}}_{0} .
\end{aligned}
$$

The last term in this expression, as well as the parallel component of $\vec{V}_{2}$, enter in at lower order when considering $\vec{\nabla}_{\perp}$ $\cdot \vec{V}_{2}$; thus they will be dropped. Including the fifth term makes it difficult to satisfy energy conservation because we do not evolve the perpendicular component of $\vec{A}_{2}$; hence, it will be dropped. The effect on linear stability of dropping this term will be discussed in Sec. IV. Even though the $\vec{\nabla}_{\perp}$ $\cdot \vec{V}_{2}$ term in the pressure and density equations are the only terms containing $\vec{V}_{2}$, it is necessary for energy conservation that the convective derivative in the pressure equation also contain $\vec{V}_{2}$; thus, the first four terms of Eq. (28) will be added to our definition of $\vec{V}_{1}$.

The definition of $\vec{V}_{1}$ gives us the salient information about the component of Ohm's Law perpendicular to the magnetic field. To obtain the information from the component of Ohm's Law along the magnetic field, the parallel component of Ohm's Law is ordered:

$O\left(\epsilon^{0}\right) \quad 0$,

$$
\begin{gathered}
O\left(\epsilon^{1}\right) \quad \vec{B}_{0} \cdot \vec{E}_{1}=-\vec{B}_{0} \cdot \vec{\nabla}_{\perp} \phi=0 \\
O\left(\epsilon^{2}\right) \quad \vec{B}_{0} \cdot \vec{E}_{2}+\vec{B}_{1} \cdot \vec{E}_{1}= \\
\quad \eta \vec{B}_{0} \cdot\left(\vec{\nabla} \times \vec{B}_{0}+\vec{\nabla}_{\perp} \times \vec{B}_{1}\right) \\
-\frac{1}{n e} \vec{B}_{0} \cdot \vec{\nabla} \cdot \Pi_{e} .
\end{gathered}
$$

The last equation can be written as an evolution equation for the parallel vector potential:

$$
\frac{\partial \Psi}{\partial t_{\|}}-\hat{\mathbf{b}}_{T} \cdot \vec{\nabla} \phi=\eta \widetilde{J_{\|}}-\frac{1}{n e} \hat{\mathbf{b}}_{0} \cdot \vec{\nabla} \cdot \Pi_{e},
$$

where $\Psi \equiv-A_{\|} \equiv-\vec{A}_{2} \cdot \hat{\mathbf{b}}_{0}$, and $\eta J_{\| 0}$ has been cancelled with $\vec{E}^{A} \cdot \hat{\mathbf{b}}_{0}+\hat{\mathbf{b}}_{0} /(n e) \cdot \vec{\nabla} \cdot \Pi_{e 0}$.

As discussed in Sec. II, the physics of fast magnetosonic waves appears in the lowest-order momentum balance. Since the goal is to eliminate these motions, an annihilator is applied to the momentum equation to eliminate the rapid motions which preserve the MHD equilibrium. The customary operator is $\vec{B} \cdot \vec{\nabla} \times$, which will yield an evolution equation for the plasma vorticity. Here we derive the vorticity equation from the quasineutrality condition, which is formally equivalent to applying the above annihilator but yields a form from which it is easier to show energy conservation. From $\vec{\nabla} \cdot \vec{J}=0$, we have

$$
(\vec{B} \cdot \vec{\nabla}) \frac{J_{\|}}{B}=-\vec{\nabla} \cdot \vec{J}_{\perp} .
$$

Ordering $\vec{J}_{\perp}$ which is obtained from the momentum equation, we find

$$
\begin{aligned}
\vec{J}_{\perp}= & \frac{\vec{B}}{B^{2}} \times\left(\rho \frac{d \vec{V}}{d t}+\vec{\nabla} p+\vec{\nabla} \cdot \Pi\right) \\
= & \frac{\vec{B}_{0} \times \vec{\nabla}_{\perp} p_{1}}{B_{0}^{2}}+\epsilon\left[\frac{\vec{B}_{1} \times \vec{\nabla}_{\perp} p_{1}}{B_{0}^{2}}+\frac{\vec{B}_{1} \times \vec{\nabla} p_{0}}{B_{0}^{2}}\right. \\
& +\frac{\vec{B}_{1} \times \vec{\nabla}_{0}}{B_{0}^{2}}\left(\frac{-2 B_{\| 1} B_{0}}{B_{0}^{2}}\right)+\frac{\rho_{0}}{B_{0}^{2}} \frac{d}{d t_{\|}} \vec{V}_{1} \\
& \left.\times \vec{B}_{0}+\frac{\vec{B}_{0}}{B_{0}^{2}} \times \vec{\nabla} \cdot \Pi\right] .
\end{aligned}
$$

The divergence of $\vec{J}_{\perp}$ is zero to leading order (this is the annihilation of the fast magnetosonic waves). In the next order, only the parallel component of $\vec{B}_{1}$ is left in the pressure term. Substituting $p_{1}=-B_{\| 1} B_{0}$, the vorticity equation becomes

$$
\begin{aligned}
\vec{\nabla} \cdot( & \left.\frac{\rho_{T}}{B_{0}} \frac{d}{d t_{\|}} \frac{\vec{\nabla} \phi}{B_{0}}\right) \\
= & \left(\overrightarrow{B_{0}} \cdot \vec{\nabla}\right) \frac{\widetilde{J_{\|}}}{B_{0}}+\left(\overrightarrow{B_{1}} \cdot \vec{\nabla}\right) \frac{J_{T_{\|}}}{B_{0}}+\vec{\nabla} \cdot \frac{\vec{B}_{0} \times \vec{\nabla} p_{1}}{B_{0}^{2}} \\
& +\vec{\nabla} \cdot \frac{p_{1}}{B_{0}^{2}} \frac{\vec{B}_{0} \times \vec{\nabla} p_{T}}{B_{0}^{2}}+\vec{\nabla} \cdot \frac{\vec{B}_{0}}{B_{0}^{2}} \times \vec{\nabla} \cdot \Pi .
\end{aligned}
$$

\section{B. Divergence of the perturbed magnetic field}

Up to this point, $\vec{B}_{1}$ has only been defined by $\vec{B}_{1}=\vec{\nabla}_{\perp}$ $\times \vec{A}_{2}$. To make the perturbed magnetic field manifestly divergence free to all orders, it is necessary to keep a lowerorder term so that $\vec{B}_{1}=\vec{\nabla} \times \vec{A}_{2}$. To relate $\vec{A}_{2}$ to the scalar quantities that are being evolved, we introduce the straightfield-line magnetic flux coordinates $\psi_{0}, \Theta, \zeta$ which are based 
upon the axisymetric equilibrium magnetic field $\vec{B}_{0}=(\vec{\nabla} \zeta$ $-q \vec{\nabla} \Theta) \times \vec{\nabla} \psi_{0}$, where $\psi_{0}$ is the poloidal flux, $\Theta$ and $\zeta$ are the poloidal and toroidal angles, respectively, and $q$ $=q\left(\psi_{0}\right)$ is the safety factor. For specificity, we express the magnetic potential using the straight-field-line coordinates in an axisymmetric magnetic field. However, one can easily generalize this treatment for a different magnetic field coordinate choice. We first express $\vec{A}_{2}$ in terms of two new scalar variables, $\psi$ (poloidal flux) and $\chi$ (toroidal flux),

$$
\vec{A}_{2}=-\psi \vec{\nabla} \zeta-\chi \vec{\nabla} \Theta,
$$

so that

$$
\vec{B}_{1}=\vec{\nabla} \zeta \times \vec{\nabla} \psi+\vec{\nabla} \Theta \times \vec{\nabla} \chi .
$$

We now need two equations to relate these new variables to the variables we are evolving. By applying $\hat{\mathbf{b}}_{0}$. to Eq. (34), $\psi$ and $\chi$ can be related to $\Psi$ by

$$
\Psi=\frac{\mathcal{J}^{-1}}{B_{0}}(q \psi+\chi),
$$

where $\mathcal{J}=\left(\vec{\nabla} \zeta \cdot \vec{\nabla} \psi_{0} \times \vec{\nabla} \Theta\right)^{-1}=\left(\vec{B}_{0} \cdot \vec{\nabla} \Theta\right)^{-1}$ is the Jacobian of our coordinate system. This relation allows one to write the perturbed magnetic field in the form

$$
\begin{aligned}
\vec{B}_{1}= & \vec{B}_{0} \frac{\partial \psi}{\partial \psi_{0}}-\mathcal{J}^{-1} R^{2} \vec{\nabla} \zeta\left(q \frac{\partial \psi}{\partial \psi_{0}}+\frac{\partial \chi}{\partial \psi_{0}}\right) \\
& +\overrightarrow{\mathcal{N}} \Theta \times \vec{\nabla} \zeta\left[\frac{\partial}{\partial \zeta}\left(\Psi B_{0}\right)-\vec{B}_{0} \cdot \vec{\nabla} \psi\right] .
\end{aligned}
$$

The next needed equation comes from the constraint required to eliminate fast magnetosonic waves, $p_{1}=-\vec{B}_{0}$ $\cdot \vec{B}_{1}$, which only needs to be satisfied to order $\epsilon: p_{1}=-\vec{B}_{0}$ $\cdot \vec{\nabla}_{\perp} \times \vec{A}_{2}$. From Eq. (37), this gives an expression for $\psi$ :

$$
\frac{\partial \psi}{\partial \psi_{0}}=-\frac{p_{1}}{B_{0}^{2}}+\frac{I}{B_{0}^{2}} \frac{\partial}{\partial \psi_{0}}\left(\Psi B_{0}\right)+\frac{q g^{\psi \Theta}}{I B_{0}^{2}} \frac{\partial}{\partial \zeta}\left(\Psi B_{0}\right),
$$

where the toroidal flux function in an axisymmetric equilibrium is $I=R B_{\text {toroidal }}$ and $g \psi \Theta=\vec{\nabla} \psi_{0} \cdot \vec{\nabla} \Theta$ is the off-diagonal metric element. When numerically solving the reduced equations, it will be necessary to numerically solve Eq. (38) for $\psi$ at each time step, which is not a serious performance issue since the equation is linear, and then algebraically solve for $\chi$ using Eq. (36) to find the perturbed magnetic field given by Eq. (35).

Also note that the method given here to obtain a divergence-free perturbed magnetic field is the first time that axisymmetry has been used in the derivation. For nonaxisymmetric configurations such as stellarators, this method can be easily generalized to an appropriate coordinate system.

This form of $\vec{B}_{1}$ is considerably more complicated than earlier representations of the perturbed magnetic field because of the necessity to keep lower order terms in $\vec{B}_{1}$. To highest order, one only needs the perpendicular component of the magnetic field: $\vec{B}_{1} \cdot \vec{\nabla} Q_{1} \approx \vec{B}_{1} \cdot \vec{\nabla}_{\perp} Q_{1}=\vec{B}_{1_{\perp}} \cdot \vec{\nabla} Q_{1}$. For high-aspect ratio reduced MHD where one expands around the toroidal magnetic field, the perpendicular magnetic field is divergence free $\left(\vec{B}_{1_{\perp}}=\vec{\nabla} \zeta \times \vec{\nabla} \psi\right)$. Also, for an expansion around a vacuum magnetic field, ${ }^{6}$ the perturbed, perpendicular magnetic field $\left(\vec{B}_{1_{\perp}}=\vec{B}_{\mathrm{vac}} \times \vec{\nabla} \psi\right)$ is divergence-free because the vacuum magnetic field is curl-free. In general, however, it is not possible to split the perturbed magnetic field into parallel and perpendicular components, and have each component also be divergence free; we solve this problem by using the form given by Eq. (35).

\section{Energy conservation}

Energy conservation is shown by multiplying Eq. (33) by $-\phi$, Eq. (23) by $V_{\|}$, and Eq. (30) by $J_{T_{\|}}$. These three equations are then added to Eq. (20) and an integration over all space is performed. Energy fluxes through the boundary of the enclosed volume are assumed to vanish. The need for keeping terms in $\vec{V}_{2}$ can be seen by looking at the third term of Eq. (23) and the fifth term of Eq. (33), both of which should cancel with the third term of Eq. 20. When forming the energy integral, only the leading order terms to $\widetilde{J_{\|}}$must be kept:

$$
\widetilde{J_{\|}}=\nabla^{2} \Psi+O(\epsilon) .
$$

With this definition of $\widetilde{J_{\|}}$, one can form a divergence term that will cancel when integrated over space and assuming no energy flow across the boundary. The integral that is conserved is

$$
\begin{array}{r}
\int d^{3} x\left(\frac{\rho_{T} V_{\|}^{2}}{2}+\frac{\rho_{T}|\vec{\nabla} \phi|^{2}}{2 B_{0}^{2}}+\frac{|\vec{\nabla} \Psi|^{2}}{2}\right. \\
\left.+\vec{\nabla} \times \vec{A}_{0} \cdot \vec{\nabla} \times\left(-\Psi \hat{\mathbf{b}}_{0}\right)+\frac{p_{1}}{\Gamma-1}\right) .
\end{array}
$$

This integral avoids the nonstandard conserved energy of the original reduced MHD derivation ${ }^{1}$ and is similar to that of Drake and Antonsen ${ }^{3}$ and Strauss. ${ }^{6}$ Using the above relations, the equations can be summarized as:

$$
\begin{aligned}
& \frac{d \rho_{1}}{d t_{\|}}+\left(\vec{V}_{1} \cdot \vec{\nabla}\right) \rho_{0}+\rho_{T} \nabla \cdot \vec{V}_{1}=0, \\
& \frac{d p_{1}}{d t_{\|}}+\left(\vec{V}_{1} \cdot \vec{\nabla}\right) p_{0}+\Gamma p_{T}\left(\nabla \cdot \vec{V}_{1}\right) \\
& \quad=(\Gamma-1)\left[\eta\left(J_{T_{\|}}^{2}+\frac{\left|\vec{\nabla} p_{T}\right|^{2}}{B_{0}^{2}}\right)-\Pi: \vec{\nabla} \vec{V}_{1}+\frac{1}{n e} \Pi_{e}: \vec{\nabla} \frac{\vec{J}}{n e}\right],
\end{aligned}
$$

$\rho_{T} \frac{d V_{\|}}{d t_{\|}}=-\hat{\mathbf{b}}_{0} \cdot \vec{\nabla} p_{1}-\hat{\mathbf{b}}_{1} \cdot \vec{\nabla} p_{T}-\hat{\mathbf{b}}_{0} \cdot \vec{\nabla} \cdot \Pi$,

$\frac{\partial \Psi}{\partial t_{\|}}-\hat{\mathbf{b}}_{T} \cdot \vec{\nabla} \phi=\eta \widetilde{J_{\|}}-\frac{1}{n e} \hat{\mathbf{b}}_{0} \cdot \vec{\nabla} \cdot \Pi_{e}$, 


$$
\begin{aligned}
\vec{\nabla} \cdot\left(\frac{\rho_{T}}{B_{0}} \frac{d}{d t_{\|}} \frac{\vec{\nabla} \phi}{B_{0}}\right) & =\left(\vec{B}_{0} \cdot \vec{\nabla}\right) \frac{\widetilde{J}_{\|}}{B_{0}}+\left(\overrightarrow{B_{1}} \cdot \vec{\nabla}\right) \frac{J_{T_{\|}}}{B_{0}}+\vec{\nabla} \cdot \frac{\vec{B}_{0} \times \vec{\nabla} p_{1}}{B_{0}^{2}} \\
& +\vec{\nabla} \cdot \frac{p_{1}}{B_{0}^{2}} \frac{\vec{B}_{0} \times \vec{\nabla} p_{T}}{B_{0}^{2}}+\vec{\nabla} \cdot \frac{\vec{B}_{0}}{B_{0}^{2}} \times \vec{\nabla} \cdot \Pi,
\end{aligned}
$$

where

$$
\begin{aligned}
\frac{d}{d t_{\|}} \equiv \frac{\partial}{\partial t_{\|}}+\left(\vec{V}_{1} \cdot \vec{\nabla}\right), \\
\vec{B}_{T} \equiv \vec{B}_{0}+\vec{B}_{1}, \quad p_{T} \equiv p_{0}+p_{1}, \quad \rho_{T} \equiv \rho_{0}+\rho_{1}, \\
J_{T_{\|}} \equiv J_{\| 0}+\widetilde{J}_{\|}=\hat{\mathbf{b}}_{0} \cdot \vec{\nabla} \times \vec{B}_{0}+\vec{\nabla}^{2} \Psi, \quad \Psi \equiv-\vec{A}_{2} \cdot \hat{\mathbf{b}}_{0}, \\
\vec{V}_{1}=\frac{\vec{B}_{0} \times \vec{\nabla} \phi}{B_{0}^{2}}+\frac{p_{1}}{B_{0}^{2}} \frac{\vec{B}_{0} \times \vec{\nabla} \phi}{B_{0}^{2}}+V_{\|} \hat{\mathbf{b}}_{T}-\eta \frac{\vec{\nabla} p_{T}}{B_{0}^{2}} \\
\quad-\frac{\vec{B}_{0} \times \vec{\nabla} \cdot \Pi_{e}}{n e B_{0}^{2}}, \\
\hat{\mathbf{b}}_{T} \equiv \hat{\mathbf{b}}_{0}+\hat{\mathbf{b}}_{1}=\frac{\vec{B}_{0}}{B_{0}}+\frac{\vec{B}_{1}}{B_{0}} .
\end{aligned}
$$

\section{Simplifications of reduced MHD equations}

The reduced MHD equations above, which have the same structure as other reduced MHD equations, have the advantage of fully reproducing the stability criterion of full MHD (see Sec. IV). Often a simplified set of equations is desired (even if the linear properties of the simplified equations are not correct) for numerical solutions where one is typically more interested in the nonlinear behavior of MHD. The equations given above can be easily simplified by making a subsidiary ordering based on $\beta \equiv p / B^{2} \sim \delta^{n}$ where $\delta$ $\ll 1$ and $n$ is a rational number. The changes due to the subsidiary ordering are primarily in the vorticity equation, pressure equation, and the definition of the velocity.

The first ordering of interest is $\beta \sim \delta^{1 / 2}$. Keeping terms of order $\delta^{1 / 2}$, but dropping terms of order $\delta$, one finds

$$
\begin{aligned}
& \frac{d \rho_{1}}{d t_{\|}}+\left(\vec{V}_{1} \cdot \vec{\nabla}\right) \rho_{0}+\rho_{T} \nabla \cdot \vec{V}_{1}=0, \\
& \frac{d p_{1}}{d t_{\|}}+\left(\vec{V}_{1} \cdot \vec{\nabla}\right) p_{0}+\Gamma p_{T}\left(\nabla \cdot \vec{V}_{1}\right) \\
& =(\Gamma-1)\left[\eta J_{T_{\|}}^{2}-\Pi: \vec{\nabla} \vec{V}+\Pi_{e}: \vec{\nabla} \frac{\vec{J}}{n e}\right], \\
& \rho_{T} \frac{d V_{\|}}{d t_{\|}}=-\hat{\mathbf{b}}_{0} \cdot \vec{\nabla} p_{1}-\hat{\mathbf{b}}_{1} \cdot \vec{\nabla} p_{T}-\hat{\mathbf{b}}_{0} \cdot \vec{\nabla} \cdot \Pi, \\
& \frac{\partial \Psi}{\partial t_{\|}}-\hat{\mathbf{b}}_{T} \cdot \vec{\nabla} \phi=\eta \widetilde{J}_{\|}-\frac{1}{n e} \hat{\mathbf{b}}_{0} \cdot \vec{\nabla} \cdot \Pi_{e},
\end{aligned}
$$

$$
\begin{aligned}
\vec{\nabla} \cdot\left(\frac{\rho_{T}}{B_{0}} \frac{d}{d t_{\|}} \frac{\vec{\nabla} \phi}{B_{0}}\right)= & \left(\vec{B}_{0} \cdot \vec{\nabla}\right) \frac{\widetilde{J_{\|}}}{B_{0}}+\left(\vec{B}_{1} \cdot \vec{\nabla}\right) \frac{J_{T_{\|}}}{B_{0}}+\vec{\nabla} \cdot \frac{\vec{B}_{0} \times \vec{\nabla} p_{1}}{B_{0}^{2}} \\
& +\vec{\nabla} \cdot \frac{\vec{B}_{0}}{B_{0}^{2}} \times \vec{\nabla} \cdot \Pi
\end{aligned}
$$

$\vec{V}_{1}=\frac{\vec{B}_{0} \times \vec{\nabla} \phi}{B_{0}^{2}}+V_{\|} \hat{\mathbf{b}}_{T}$.

The energy integral that is conserved here is the same as the full set of reduced equations, but we note that it is not possible to show that the electron stress tensor term in the pressure equation is positive definite in this case. The equations in this order still retain sound waves, but are simplified primarily in the definition of $\vec{V}_{1}$, and the elimination of one of the curvature terms in the vorticity equation. The equations here are similar to the equations recently derived by Strauss. ${ }^{6}$

The next ordering of interest is $\beta \sim \delta$. After eliminating terms of order $\delta$, the relevant equations are

$$
\begin{aligned}
& \frac{d p_{1}}{d t_{\|}}+\left(\vec{V}_{1} \cdot \vec{\nabla}\right) p_{0}=0, \\
& \frac{\partial \Psi}{\partial t_{\|}}-\hat{\mathbf{b}}_{T} \cdot \vec{\nabla} \phi=\eta \widetilde{J_{\|}}-\frac{1}{n e} \hat{\mathbf{b}}_{0} \cdot \vec{\nabla} \cdot \Pi_{e}, \\
& \vec{\nabla} \cdot\left(\frac{\rho_{T}}{B_{0}} \frac{d}{d t_{\|}} \frac{\vec{\nabla} \phi}{B_{0}}\right)=\left(\vec{B}_{0} \cdot \vec{\nabla}\right) \frac{\widetilde{J_{\|}}}{B_{0}}+\left(\vec{B}_{1} \cdot \vec{\nabla}\right) \frac{J_{T_{\|}}}{B_{0}} \\
& +\vec{\nabla} \cdot \frac{\vec{B}_{0} \times \vec{\nabla} p_{1}}{B_{0}^{2}}+\vec{\nabla} \cdot \frac{\vec{B}_{0}}{B_{0}^{2}} \times \vec{\nabla} \cdot \Pi, \\
& \vec{V}_{1}=\frac{\vec{B}_{0} \times \vec{\nabla} \phi}{B_{0}^{2}},
\end{aligned}
$$

where the curvature term is ordered $\delta^{1 / 2}$. Eliminating the divergence term in the pressure equation at this level eliminates sound waves. Because the divergence term is eliminated, it is also possible to keep only the highest order term in the convective derivative, $\vec{V}_{\perp} \cdot \vec{\nabla}$, and still satisfy energy conservation. In this case, which we choose for simplicity, the parallel momentum equation becomes decoupled from the other equations leaving only four variables that need to be evolved $(\rho, p, \phi, \Psi)$. If it is assumed that the divergence term in the density equation can be eliminated at this order (or that $V_{\|}$is chosen such that this term is eliminated), and that the density is constant in time and space, then energy conservation can be satisfied without the density equation and only three variables need to be evolved $(p, \phi, \Psi)$. This form of the equations are similar to conventional "high- $\beta$ ", reduced MHD, and like conventional reduced MHD, the energy conservation relation that it satisfies is non-standard ${ }^{2}$ due to the elimination of sound waves. The explicit derivation of conventional reduced MHD from these equations is shown in Appendix B.

The final ordering of interest is $\beta \sim \delta^{2}$. The relevent equations at this order are 


$$
\begin{aligned}
& \frac{\partial \Psi}{\partial t_{\|}}-\hat{\mathbf{b}}_{T} \cdot \vec{\nabla} \phi=0, \\
& \vec{\nabla} \cdot\left(\frac{\rho_{T}}{B_{0}} \frac{d}{d t_{\|}} \frac{\vec{\nabla} \phi}{B_{0}}\right)=\left(\vec{B}_{0} \cdot \vec{\nabla}\right) \frac{\widetilde{J_{\|}}}{B_{0}}+\left(\vec{B}_{1} \cdot \vec{\nabla}\right) \frac{J_{T_{\|}}}{B_{0}} \\
& +\vec{\nabla} \cdot \frac{\vec{B}_{0}}{B_{0}^{2}} \times \vec{\nabla} \cdot \Pi \\
& \vec{V}_{1}=\frac{\vec{B}_{0} \times \vec{\nabla} \phi}{B_{0}^{2}} .
\end{aligned}
$$

This ordering eliminates the curvature term in the vorticity equation which decouples the pressure equation from the other equations leaving two variables to be evolved $(\phi, \Psi)$. This set most closely resembles the conventional 'low- $\beta$ ', reduced MHD equations. ${ }^{1}$

\section{E. Equilibrium shear flow}

Here we briefly show that it is possible to selfconsistently add equilibrium shear flow to the previous equations. To do so, we change the velocity ordering in Eq. (8) to

$$
\vec{V}=\epsilon \vec{V}_{0}+\epsilon \vec{V}_{1} \text {. }
$$

The equilibrium flow velocity is ordered such that

$$
\begin{aligned}
& \frac{\vec{B}_{0}}{\sqrt{\rho \mu_{0}}} \cdot \vec{\nabla} \sim \vec{V}_{0} \cdot \vec{\nabla}_{\perp}, \\
& \frac{V_{0}}{V_{A}} \sim \frac{k_{\|}}{k_{\perp}} \sim \epsilon .
\end{aligned}
$$

Equation (60) is motivated by stability studies, while the equivalent scaling of Eq. (61) shows that we are using a low Alfven Mach number approximation, which is valid for magnetic fusion plasma experiments. Because of this ordering, terms that contain only equilibrium quantities will appear in lower-order equations. Without loss of consistency, we are able to assume $\partial \vec{V}_{0} / \partial t=0$ and add these terms to the equilibrium equations [Eq. (13)] so that they become:

$$
\begin{aligned}
& \vec{\nabla} \cdot \rho_{0} \vec{V}_{0}=0, \\
& \vec{V}_{0} \cdot \vec{\nabla} p_{0}+\Gamma p_{0}\left(\vec{\nabla} \cdot \vec{V}_{0}\right)=0, \\
& \vec{\nabla} \times\left(\vec{V}_{0} \times \vec{B}_{0}\right)=0, \\
& \rho_{0}\left(\vec{V}_{0} \cdot \vec{\nabla}\right) \vec{V}_{0}=-\vec{\nabla}\left(p_{0}+B_{0}^{2} / 2\right)+\left(\vec{B}_{0} \cdot \vec{\nabla}\right) \vec{B}_{0} .
\end{aligned}
$$

These are the equilibrium equations with shear flow that were studied by Hameiri. ${ }^{14}$ As shown in that reference, the equilibrium shear flow may be written in the form

$$
\vec{V}_{0}=\frac{\vec{B}_{0} \times \vec{\nabla}_{\perp} \phi_{0}}{B_{0}^{2}}+V_{0_{\|}} \hat{\mathbf{b}}_{0},
$$

where $\phi_{0}=\phi_{0}\left(\psi_{0}\right)$. Because it is possible to write the equilibrium flow velocity field in the same form as the perturbed velocity field, one can add shear flow to Eqs. (41) by changing $\phi$ to $\phi_{0}+\phi$ and dropping any equilibrium terms. In con- trast to conventional reduced MHD (where $\vec{V}_{1}=\vec{\nabla} \zeta \times \vec{\nabla} \phi$ ), these equations allow for flows other than a poloidal flow.

\section{F. Closure relations}

Stress tensor terms are ignored in the resistive MHD approximation. In this section, we briefly discuss the stress tensor terms included in these equations in terms of neoclassical closure theory. ${ }^{15,16}$ In the neoclassical MHD theory ${ }^{7}$ for a tokamak plasma, the differences between the parallel (perpendicular) and toroidal (poloidal) directions is crucial for obtaining the key physics results. For conventional reduced MHD where there is no such distinction, it is difficult to incorporate all of the relevent physics. ${ }^{7,17}$ However, in the present formulation, the neoclassical physics is easily captured.

The vorticity and momentum balance equations require different forms of the viscous stress tensors. Unfortunately, conventional neoclassical theory only yields particular projections of the stress tensors in particular asymptotic temporal regimes (namely on time scales long compared to the ion collision time). In this work, we propose a form for the viscous stress tensor which yields the correct asymptotic forms. Namely, for $\vec{\nabla} \cdot \Pi$, we suggest the form

$$
\vec{\nabla} \cdot \Pi_{s}=\rho_{s} \mu_{s}\left\langle B^{2}\right\rangle \frac{\vec{V}_{s} \cdot \vec{\nabla} \Theta}{(\vec{B} \cdot \vec{\nabla} \Theta)^{2}} \vec{\nabla} \Theta,
$$

where $\mu_{s}$ is the viscous damping frequency for each species $s$, which depends on the collisionality regime.

This form for the ion viscous stress tensor yields the important physics of poloidal flow damping from the parallel momentum balance equation. It is important to realize that this effect cannot be represented in conventional reduced MHD descriptions since the only perturbed flow allowed within the flux surface is poloidal. ${ }^{17}$ Further, the presence of a viscous term in the vorticity equation in combination with the parallel momentum balance gives the neoclassical polarization-current enhancement of neoclassical MHD theory. ${ }^{7}$

For the perturbed electron stress tensor, the form is simplified if we order the relation between the electron velocity $\vec{V}_{e}$ and the MHD variables that we are evolving:

$$
\vec{V}_{e_{1}}=\vec{V}-\frac{\vec{J}}{n e}=-\frac{1}{n e}\left(\widetilde{J}_{\|} \hat{\mathbf{b}}_{0}+\frac{\vec{B}_{0} \times \vec{\nabla} p_{1}}{B_{0}^{2}}\right)+O(\epsilon) .
$$

This form of the electron velocity when substituted into the electron stress tensor term in the parallel Ohm's Law gives rise to the neoclassical enhancement of the plasma resistivity and the perturbed bootstrap current, which is important for neoclassical tearing modes.

The suggested forms for the viscous stress terms have convenient forms for demonstrating energy conservation. The stress tensor terms on the right-hand side of Eqs. (23), (30), and (33) can be shown to be negative definite thus heating the plasma through the viscous heating terms that appear in the pressure equation with which they cancel. 


\section{LINEAR STABILITY ANALYSIS OF THE REDUCED EQUATIONS}

Writing the equilibrium magnetic field as $\vec{B}_{0}=\vec{\nabla} \alpha$ $\times \vec{\nabla} \psi$, the perpendicular wave number can be written as $k_{\perp}=k_{\psi} \vec{\nabla} \psi+k_{\alpha} \vec{\nabla} \alpha$. This expression for $k_{\perp}$ allows us to classify modes for which our ordering is valid into two categories: (1) resistive layer modes, such as interchange and tearing modes, where the modes localize near a rational surface and one can order

$$
k_{\psi} \sim \frac{1}{\epsilon}, \quad k_{\alpha} \sim 1 ;
$$

and (2) ballooning modes where both $k_{\psi}$ and $k_{\alpha}$ are order $1 / \epsilon$ such that the modes are poloidally localized. Applying the WKB formalism of ballooning theory to our equations reproduces the ballooning equation in a manner that is similar to the treatment of Hazeltine and Meiss. ${ }^{5}$ In this section, we discuss the resistive layer modes in a general axisymetric device.

\section{A. Resistive layer modes}

In this section, the inner linear resistive-layer equations for the reduced equations are discussed. The layer equations are derived from the narrow layer width approximation characterized by the ordering

$$
x \equiv \psi-\psi_{s} \sim \gamma \sim \eta^{1 / 3} \sim \epsilon, \quad \frac{\partial}{\partial \psi_{0}} \sim \frac{1}{\epsilon} .
$$

A detailed derivation of the inner-layer equations, shown in Appendix C, follows the methods used by Johnson and Green $^{18}$ and Glasser, Greene, and Johnson ${ }^{19}$ (GGJ). The results are contrasted with GGJ to elucidate the approximations that were made in the derivation of the reduced equations.

Neglecting the stress-tensor contributions, the innerlayer equations become

$$
\begin{aligned}
& \bar{\Psi}_{X X}-H \Upsilon_{X}=Q(\bar{\Psi}-X \Xi), \\
& Q^{2} \Xi_{X X}-Q X^{2} \Xi+E \Upsilon+Q X \Psi+\Gamma=0, \\
& Q \Upsilon_{X X}-X^{2} \Upsilon-Q^{2} G \Upsilon+(G-K E-R) Q^{2} \Xi+X \bar{\Psi} \\
& \quad-K Q^{2} \Gamma=0, \\
& \Gamma_{X}=H \bar{\Psi}_{X X}+F \Upsilon_{X},
\end{aligned}
$$

for the variables $\bar{\Psi}, \Xi, \Upsilon$, and $\Gamma$. The definitions of the variables and constants may be found in Appendix C.

The equations exactly match those of GGJ except for the term in the pressure equation containing $R$. Using the definition given in Appendix $\mathrm{C}$, it is possible to express $R$ as

$$
R=\frac{\left\langle B_{0}^{2}\right\rangle\left\langle 1 / B_{0}^{2}\right\rangle}{\left\langle B_{0}^{2} B_{T}^{2} / B_{P}^{2}\right\rangle\left[\left\langle B_{P}^{2} / B_{T}^{2} B_{0}^{2}\right\rangle+\left\langle 1 / B_{0}^{2}\right\rangle-1 /\left\langle B_{0}^{2}\right\rangle\right]},
$$

where $B_{T}\left(B_{P}\right)$ is the toroidal (poloidal) magnetic field. As can be seen, this term is of order unity, which will be smaller than $G$ when $G$ is large enough to be significant as shown in the Appendix of GGJ. Hence, the reduced equations presented here exactly reproduce the ideal and resistive MHD instability criterion and growth rates.

We conclude that the dropping of the term in our equation for $\vec{V}_{2}$, which is not rigorously justified in our expansion yet necessary for energy conservation, has negligible effects on the linear stability. Our other significant approximation, $\widetilde{J_{\|}}=\nabla^{2} \Psi$, also has no effect on stability. The reduced MHD description given in this work reproduces the linear growth rates and instability conditions for tearing modes in a tokamak plasma because the narrow-layer width ordering is a subset of the ordering used in these equations.

\section{SUMMARY}

A new multiple-time-scale technique based on assuming a disparity of wavelengths along and perpendicular to the magnetic field has been used to reduce the MHD equations. This derivation has the advantage of clearly separating the three time scales of the problem associated with (1) MHD equilibrium, (2) fluctuations whose wave vector is aligned perpendicular to, and (3) those aligned parallel to the magnetic field. On the equilibrium time scale, the equilibrium quantities were shown to be time independent and satisfy exactly the MHD equilibrium equations, in contrast with conventional reduced MHD where the equilibrium equations themselves are reduced versions of the general equilibrium equations. The fast, perpendicular time-scale motions, which were shown to be fast magnetosonic waves, came in at the same order as the equilibrium equations. To assume that these waves equilibrate to the equilibrium, constraints must be placed on the perturbed quantities. These constraints allow one to derive equations that evolve five scalar functions on a time scale associated with the parallel wave vector (shear-Alfven wave time scale), which is the time scale of interest for instability studies.

A major advantage of the reduced equations derived here is that they reproduce the stability criterion for resistive layer modes. Because the perturbations are expanded about the exact equilibrium equations, the derivation followed in this work is similar to previous linear stability analyses. ${ }^{11,18-20}$ However, by emphasizing energy conservation and a divergence-free perturbed magnetic field when keeping the nonlinear terms, these equations are suitable for nonlinear, numerical simulations. Conventional reduced MHD, which does not contain the correct linear physics, has been a successful model for numerical simulations of plasma phenomena because it contains the key nonlinear physics and maintains an energy conservation relation. The equations here incorporate results learned from linear stability analysis within the framework of reduced MHD.

The reduced MHD equations derived here also allow the consideration of two effects not normally considered in reduced MHD: equilibrium shear flow and neoclassical effects. The inclusion of both of these effects is aided by keeping the evolution equation for the parallel velocity, which is normally not evolved. The inclusion of the parallel velocity allows the equilibrium velocity to be expressed in the same form as the first-order velocity so that inclusion of shear-flow 
effects is very straightforward. The keeping of the parallel evolution equation allows the neoclassical effects of poloidal flow damping and polarization current enhancement to be included in addition to the bootstrap current which had been used in reduced equations previously. ${ }^{17}$ The numerical solution of these equations, including these two effects, will be considered in future work.

\section{ACKNOWLEDGMENTS}

The authors wish to thank H.R. Strauss and R.L. Dewar for pointing out their useful references, and T.A. Gianakon for help in expressing the energy conservation integral in a nice form. We also wish to thank Dr. John M. Greene for providing his Lausanne lecture notes, and for valuable discussions concerning our stability analysis of the reduced equations. This research is supported by the United States Department of Energy (DOE) under Grant Nos. DE-FG0286ER53218 and DE-FG02-92ER54139.

\section{APPENDIX A: PROPERTIES OF THE PERPENDICULAR AND PARALLEL GRADIENT OPERATORS}

The ordering used is somewhat unusual in that it is based on operators rather than physical quantities. Because the derivation requires a familiarity with the properties of the $\vec{\nabla}_{\|}$ and $\vec{\nabla}_{\perp}$ operators, this appendix summarizes those that were found useful.

We define

$$
\begin{aligned}
& \vec{\nabla}_{\|} \equiv \hat{\mathbf{b}}_{0}\left(\hat{\mathbf{b}}_{0} \cdot \vec{\nabla}\right), \\
& \vec{\nabla}_{\perp} \equiv \vec{\nabla}-\hat{\mathbf{b}}_{0}\left(\hat{\mathbf{b}}_{0} \cdot \vec{\nabla}\right),
\end{aligned}
$$

and consider the operations of these operators on any scalar function $f$ and vector function $\vec{A}$.

\section{Gradient}

$$
\begin{aligned}
& \vec{\nabla}_{\|} f=\hat{\mathbf{b}}_{0}\left(\hat{\mathbf{b}}_{0} \cdot \vec{\nabla}\right) f, \\
& \vec{\nabla}_{\perp} f=\vec{\nabla} f-\hat{\mathbf{b}}_{0}\left(\hat{\mathbf{b}}_{0} \cdot \vec{\nabla}\right) f,
\end{aligned}
$$

so that

$$
\begin{aligned}
& \vec{B}_{0} \cdot \vec{\nabla}_{\perp} f=0, \\
& \vec{B}_{0} \cdot \vec{\nabla}_{\|} f=\vec{B}_{0} \cdot \vec{\nabla} f .
\end{aligned}
$$

\section{Divergence}

$$
\begin{aligned}
\vec{\nabla}_{\|} \cdot \vec{A} & =\hat{\mathbf{b}}_{0}\left(\hat{\mathbf{b}}_{0} \cdot \vec{\nabla}\right) \cdot \vec{A}, \\
& =\left(\hat{\mathbf{b}}_{0} \cdot \vec{\nabla}\right) \vec{A} \cdot \hat{\mathbf{b}}_{0}-\vec{A} \cdot\left(\hat{\mathbf{b}}_{0} \cdot \vec{\nabla}\right) \hat{\mathbf{b}}_{0}, \\
& =\hat{\mathbf{b}}_{0} \cdot \vec{\nabla} A_{\|}-\vec{A} \cdot \overrightarrow{\boldsymbol{\kappa}},
\end{aligned}
$$

where the curvature vector is defined by

$$
\vec{\kappa} \equiv\left(\hat{\mathbf{b}}_{0} \cdot \vec{\nabla}\right) \hat{\mathbf{b}}_{0} .
$$

The perpendicular divergence is then

$$
\vec{\nabla}_{\perp} \cdot \vec{A}=\vec{\nabla} \cdot \vec{A}-\hat{\mathbf{b}}_{0} \cdot \vec{\nabla} A_{\|}+\vec{A} \cdot \vec{\kappa}
$$

These relations are identities. Because $\lambda_{\perp} \ll a$, the gradients of the equilibrium quantities are order $\epsilon$ smaller than the perpendicular gradients of perturbed quantities and can cause confusion when ordering these relations. As a simple example, consider the divergence of $A_{\|} \hat{\mathbf{b}}_{0}$. To first order we have the perpendicular divergence:

$$
\vec{\nabla}_{\perp} \cdot A_{\|} \hat{\mathbf{b}}_{0}=\hat{\mathbf{b}}_{0} \cdot \vec{\nabla}_{\perp} A_{\|}+A_{\|} \vec{\nabla}_{\perp} \cdot \hat{\mathbf{b}}_{0} .
$$

The first term is zero and the second term should really be interpreted as $\vec{\nabla} \cdot \hat{\mathbf{b}}_{0}$, which is order $\epsilon$. Thus, this last term appears in the next order of the divergence, which is the parallel divergence:

$$
\vec{\nabla}_{\|} \cdot A_{\|} \hat{\mathbf{b}}_{0}=\hat{\mathbf{b}}_{0} \cdot \vec{\nabla}_{\|} A_{\|}+A_{\|} \vec{\nabla} \cdot \hat{\mathbf{b}}_{0} .
$$

As a practical way of deriving many of the terms, one can take the $\vec{\nabla}_{\perp}$ operation on an equilibrium quantity to be zero, and convert $\vec{\nabla}_{\|}$operating on an equilibrium quantity to be $\vec{\nabla}$, which is similar to how Hazeltine and Meiss ${ }^{5}$ present their derivation. The fundamental ordering however is that given by Eq. (2).

\section{Curl}

$$
\begin{aligned}
\vec{\nabla}_{\|} \times \vec{A} & =\hat{\mathbf{b}}_{0}\left(\hat{\mathbf{b}}_{0} \cdot \vec{\nabla}\right) \times \vec{A} \\
& =\left(\hat{\mathbf{b}}_{0} \cdot \vec{\nabla}\right) \hat{\mathbf{b}}_{0} \times \vec{A}-\vec{A} \times\left(\hat{\mathbf{b}}_{0} \cdot \vec{\nabla}\right) \hat{\mathbf{b}}_{0}, \\
& =\nabla_{\|}\left(\hat{\mathbf{b}}_{0} \times \vec{A}\right)-\vec{A} \times \overrightarrow{\boldsymbol{\kappa}}, \\
& =\left(\vec{B}_{0} \cdot \vec{\nabla}\right) \frac{\vec{B}_{0} \times \vec{A}}{B_{0}^{2}}-\hat{\mathbf{b}}_{0} \times \vec{A} \nabla_{\|} \ln \left(B_{0}\right)-\vec{A} \times \overrightarrow{\boldsymbol{\kappa}} .
\end{aligned}
$$

The perpendicular curl is

$$
\vec{\nabla}_{\perp} \times \vec{A}=\vec{\nabla} \times \vec{A}_{\perp}-\vec{\nabla}_{\|} \times \vec{A} .
$$

Note that $\vec{B}_{0} \cdot \vec{\nabla}_{\|} \times \vec{A}=0$.

\section{APPENDIX B: REDUCTION OF EQUATIONS TO CONVENTIONAL REDUCED MHD}

In this Appendix, the relation of Eqs. (46), where it has already been assumed that $\beta$ is small, to conventional reduced MHD is made explicit. Conventional reduced MHD uses the large-aspect ratio assumption of

$$
\epsilon \equiv \frac{a}{R}, \quad \frac{B_{\text {poloidal }}}{B_{\text {toroidal }}} \sim \epsilon .
$$

As shown in Eq. (1), this ordering is a subset of the ordering already used. The dominant change in Eqs. (46) is due to the equilibrium magnetic field also being ordered. The parallel direction is now the toroidal direction:

$$
\hat{\mathbf{b}}_{0}=R_{0} \vec{\nabla} \zeta .
$$

The toroidal flux function $I$ is approximately constant in this ordering, such that the magnitude of the magnetic field is also a constant $\left(B_{0} \approx I_{0} / R_{0}\right)$. 
Only the perpendicular component of the perturbed magnetic field is kept because, as discussed in Sec. III, to highest order $\vec{B}_{1} \cdot \vec{\nabla} \approx \vec{B}_{1} \cdot \vec{\nabla}_{\perp}=\vec{B}_{1_{\perp}} \cdot \vec{\nabla}$. The perpendicular component of the magnetic field is, using Eq. (35),

$$
\vec{B}_{1_{\perp}}=\vec{\nabla} \zeta \times \vec{\nabla} \psi
$$

Similarly, the expression for the velocity in Eq. (46) becomes

$$
\vec{V}_{1_{\perp}}=\frac{\vec{B}_{0} \times \vec{\nabla} \phi}{B_{0}^{2}}=\vec{\nabla} \zeta \times \vec{\nabla} \phi^{\prime}
$$

where $\phi^{\prime}=\left(R_{0}^{2} / I_{0}\right) \phi$. Like the perpendicular magnetic field, the perpendicular velocity field is divergence-free in this model.

We first consider the pressure equation. In this paper, a distinction was made between equilibrium terms and perturbed quantities because the ordering was performed differently for the two quantities and the equilibrium equation was not reduced; however, this distinction is usually not made for reduced equations where $p$ denotes both the equilibrium and perturbed quantities. Ignoring the stress tensors and resistive heating, the pressure equation becomes

$$
\frac{\partial p}{\partial t_{\|}}+\vec{\nabla} \zeta \cdot \vec{\nabla} \phi^{\prime} \times \vec{\nabla} p=0 .
$$

Ohm's Law becomes

$$
\frac{\partial \psi}{\partial t_{\|}}-I \vec{\nabla} \zeta \cdot \phi^{\prime}-\vec{\nabla} \zeta \cdot \vec{\nabla} \psi \times \vec{\nabla} \phi^{\prime}=\eta \vec{\nabla}^{2} \psi,
$$

where the parallel vector potential has been related to the poloidal flux by ordering the parallel component of Eq. (34):

$$
\Psi \approx \frac{\psi}{R_{0}}
$$

At high-aspect ratio, where only the perpendicular gradient is needed and the magnetic field is approximately constant, the inertia term in the vorticity equation becomes

$$
\frac{1}{I_{0}}\left(\rho_{0} \frac{\partial U}{\partial t_{\|}}+\rho_{0} \vec{\nabla} \zeta \cdot \vec{\nabla} \phi^{\prime} \times \vec{\nabla} U\right),
$$

where $U=\vec{\nabla}_{\perp}^{2} \phi^{\prime}$. The curvature term becomes

$$
\vec{\nabla} p \cdot \vec{\nabla} \times \frac{\vec{B}_{0}}{B_{0}^{2}}=-\frac{2}{I_{0}} \vec{\nabla} \zeta \times \vec{\nabla} R \cdot \vec{\nabla} p .
$$

For the kink terms, we first write the equilibrium parallel current as $\vec{\nabla}_{\perp}^{2} \psi_{0}$. Again we do not distinguish between equilibrium and perturbed quantities to write the kink terms as

$$
\vec{\nabla} \zeta \cdot \vec{\nabla} J_{\|}+\frac{1}{I_{0}} \vec{\nabla} \zeta \cdot \vec{\nabla} \psi \times \vec{\nabla} J_{\|} \cdot
$$

Multiplying through by $I_{0}$, the vorticity equation becomes

$$
\rho_{0} \frac{d U}{d t_{\|}}=I_{0} \vec{\nabla} \zeta \cdot \vec{\nabla} J_{\|}+\vec{\nabla} \zeta \cdot \vec{\nabla} \psi \times \vec{\nabla} J_{\|}-2 \vec{\nabla} \zeta \times \vec{\nabla} R \cdot \vec{\nabla} p,
$$

where

$$
U \equiv \vec{\nabla}_{\perp}^{2} \phi^{\prime}, \quad J_{\|} \equiv \vec{\nabla}_{\perp}^{2} \psi, \quad \frac{d}{d t_{\|}} \equiv \frac{\partial}{\partial t_{\|}}+\vec{\nabla} \zeta \cdot \vec{\nabla} \phi^{\prime} \times \vec{\nabla} .
$$

Equations (B5), (B6), and (B11) are the conventional reduced MHD equations.

\section{APPENDIX C: DERIVATION OF THE RESISTIVE INNER-LAYER EQUATIONS}

In this Appendix, linear equations are found for the inner resistive layer based on the narrow-layer width approximation. The formalism used in Refs. $18-20$ is used in deriving these equations.

We begin with the linearized version of Eqs. (41) with the perturbed quantities now denoted with tildes:

$$
\begin{aligned}
& \rho \frac{\partial \widetilde{V_{\|}}}{\partial t}=-\hat{\mathbf{b}}_{0} \cdot \vec{\nabla} \tilde{p}-\frac{\widetilde{B}}{B_{0}} \cdot \vec{\nabla} p_{0}, \\
& \frac{\partial \widetilde{\Psi}}{\partial t}-\hat{\mathbf{b}}_{0} \cdot \vec{\nabla} \widetilde{\phi}=\eta \vec{\nabla}^{2} \widetilde{\Psi}, \\
& \vec{\nabla} \cdot \frac{\rho}{B_{0}^{2}} \frac{\partial \vec{\nabla} \tilde{\phi}}{\partial t}=\vec{\nabla} \cdot \frac{\vec{B}_{0} \times \vec{\nabla} \tilde{p}}{B_{0}^{2}}+\vec{\nabla} \cdot \frac{\tilde{p}}{B_{0}^{2}} \frac{\vec{B}_{0} \times \vec{\nabla} p_{0}}{B_{0}^{2}} \\
& +\vec{B}_{0} \cdot \vec{\nabla} \frac{\widetilde{J_{\|}}}{B_{0}}+(\widetilde{\vec{B}} \cdot \vec{\nabla}) \frac{J_{\| 0}}{B_{0}},
\end{aligned}
$$

$$
\frac{\partial \tilde{p}}{\partial t}+\frac{\vec{B}_{0} \times \vec{\nabla} \widetilde{\phi}}{B_{0}^{2}} \cdot \vec{\nabla} p_{0}+\Gamma p_{0} \vec{\nabla} \cdot \tilde{\vec{V}}=0
$$

We will consider a narrow-layer width at the $q=M / N$ rational surface where we adopt the usual resistive layer ordering $^{18-20}$

$$
x \equiv \psi-\psi_{s} \sim \gamma \sim \eta^{1 / 3} \sim \epsilon, \quad \frac{\partial}{\partial \psi_{0}} \sim \frac{1}{\epsilon} .
$$

Equilibrium quantities will be considered to be approximately constant across the layer. We also order the perturbed variables as:

$$
\tilde{p}, \widetilde{V_{\|}} \sim 1, \widetilde{\phi}, \widetilde{\Psi}, \widetilde{\psi} \sim \epsilon .
$$

The potentials are ordered such that when the derivative with respect to $\psi_{0}$ is taken, they will be of the same order as the primitive variables.

Because we are considering perturbed quantities near the rational surface, it is helpful to change from the straightfield-line coordinate system of Sec. III (using $\psi_{0}, \Theta, \zeta$ ) to a $\psi_{0}, \Theta, u$ coordinate system where $\mathrm{u}$ is the helical angle defined as $u \equiv N \zeta-M \Theta$. This translation of coordinates will allow easier identification of resonant perturbations when Fourier expanding the perturbed quantities: 


$$
\begin{aligned}
\widetilde{Q} & =\underbrace{\sum_{m, n} Q_{m, n}\left(\psi_{0}\right) e^{i(m \Theta-n \zeta)} e^{i \gamma t}}_{\text {resonant perturbation }} \\
= & \underbrace{Q_{M, N}\left(\psi_{0}\right) e^{-i u} e^{i \gamma t}}_{\text {non-resonant perturbation }} \\
& +\sum_{m \neq n, N} Q_{m, n}\left(\psi_{0}\right) e^{-i(n / N) u} e^{i n \Theta(m / n-M / N)} e^{i \gamma t} \\
= & \sum_{n} Q_{n}\left(\psi_{0}, \Theta\right) e^{i \gamma t-i u} .
\end{aligned}
$$

The last line is the procedure we will adopt for simplicity. If after transforming, we find $\widetilde{Q}$ to be independent of $\Theta$, then only the resonant harmonic is present. The helical coordinate also facilitates the representation of the parallel gradient operator:

$$
\begin{aligned}
\vec{B}_{0} \cdot \vec{\nabla} & =\mathcal{J}^{-1}\left[\frac{\partial}{\partial \Theta}-(M-N q) \frac{\partial}{\partial u}\right], \\
& \approx \mathcal{J}^{-1} \frac{\partial}{\partial \Theta}-\epsilon i N \Lambda \frac{V^{\prime} \mathcal{J}^{-1}}{4 \pi^{2}} x,
\end{aligned}
$$

where $\Lambda=4 \pi^{2} q^{\prime} / V^{\prime}, x=\psi-\psi_{s}$ where $q\left(\psi_{s}\right)=M / N, V$ is the volume enclosed by the flux surface, and primes denote derivatives with respect $\psi_{0}$. In the last line, $M-N q$ was Taylor expanded about the rational surface after Fourier expanding. Also note that for convenience the Jacobian here refers to the original Jacobian in the $\psi_{0}, \Theta, \zeta$ coordinate system which differs from the new Jacobian by a factor of $N .^{20}$

Before considering the ordered equations, first consider common operations on perturbed quantities. We begin by writing

$$
\vec{\nabla} \widetilde{Q}=\frac{\partial \widetilde{Q}}{\partial \psi_{0}} \vec{\nabla} \psi_{0}+\widetilde{Q}_{\wedge} \vec{B}_{0} \times \vec{\nabla} \psi_{0}+\widetilde{Q}_{\|} \hat{\mathbf{b}}_{0},
$$

where the cross-field derivative coefficient $\widetilde{Q}_{\wedge}$ is

$$
\widetilde{Q}_{\wedge}\left|\vec{\nabla} \psi_{0}\right|^{2}=i N \widetilde{Q}+\frac{I}{B_{0}^{2}} \vec{B}_{0} \cdot \vec{\nabla} \widetilde{Q} .
$$

This allows us to write

$$
\frac{\vec{B}_{0} \times \vec{\nabla} \widetilde{Q}}{B_{0}^{2}}=-\widetilde{Q} \wedge \vec{\nabla} \psi_{0}+\frac{\partial \widetilde{Q}}{\partial \psi_{0}} \frac{\vec{B}_{0} \times \vec{\nabla} \psi_{0}}{B_{0}^{2}} .
$$

The divergence of this quantity is

$$
\begin{aligned}
\vec{\nabla} \cdot \frac{\vec{B}_{0} \times \vec{\nabla} \widetilde{Q}}{B_{0}^{2}}=-\frac{\partial \widetilde{Q}}{\partial \psi_{0}}\left(\vec{B}_{0} \cdot \vec{\nabla}\right) \frac{J_{\| 0}}{p_{0}^{\prime} B_{0}} \\
+\widetilde{Q}_{\wedge}\left|\vec{\nabla} \psi_{0}\right|^{2}\left(2 \kappa_{\psi}-\frac{p_{0}^{\prime}}{B_{0}^{2}}\right)+\frac{J_{\| 0} / B_{0}}{B_{0}^{2}} \vec{B}_{0} \cdot \vec{\nabla} \widetilde{Q},
\end{aligned}
$$

where we have used

$$
\vec{\kappa}=\kappa_{\psi} \frac{\vec{\nabla} \psi_{0}}{\left|\vec{\nabla} \psi_{0}\right|^{2}}+\kappa \wedge \vec{B}_{0} \times \vec{\nabla} \psi_{0}
$$

$$
2 \kappa \wedge\left|\vec{\nabla} \psi_{0}\right|^{2}=-\frac{\vec{B}_{0} \times 2 \vec{\kappa}}{B_{0}^{2}} \cdot \vec{\nabla} \psi_{0}=\vec{B}_{0} \cdot \vec{\nabla} \frac{J_{\| 0}}{p_{0}^{\prime} B_{0}} .
$$

The first term on the right hand side of Eq. (C10) will be the highest-order term in that expression.

With these relations and definitions, the ordering of Eqs. (C1) is simplified. The parallel momentum balance, parallel Ohm's Law, vorticity equation, and pressure equation are to lowest order

$\mathcal{J}^{-1} \frac{\partial \tilde{p}}{\partial \Theta}=0$,

$\mathcal{J}^{-1} \frac{\partial \widetilde{\phi}}{\partial \Theta}=0$

$\mathcal{J}^{-1} \frac{\partial}{\partial \Theta}\left(\frac{\partial^{2}}{\partial \psi_{0}^{2}} \frac{\tilde{\Psi} B_{0}\left|\vec{\nabla} \psi_{0}\right|^{2}}{B_{0}^{2}}\right)-\frac{\partial \tilde{p}}{\partial \psi_{0}} \mathcal{J}^{-1} \frac{\partial}{\partial \Theta}\left(\frac{J_{\| 0}}{p_{0}^{\prime} B_{0}}\right)=0$

$\mathcal{J}^{-1} \frac{\partial}{\partial \Theta}\left(\frac{\widetilde{V_{\|}} B_{0}}{B_{0}^{2}}\right)-\frac{\partial \widetilde{\phi}}{\partial \psi_{0}} \mathcal{J}^{-1} \frac{\partial}{\partial \Theta}\left(\frac{J_{\| 0}}{p_{0}^{\prime} B_{0}}\right)=0$,

and in the next order

$$
\begin{aligned}
\gamma \rho \widetilde{V_{\|}} B_{0}= & -\mathcal{J}^{-1} \frac{\partial \widetilde{p}^{(2)}}{\partial \Theta}+i N \Lambda x \frac{V^{\prime} \mathcal{J}^{-1}}{4 \pi^{2}} \tilde{p}+p_{0}^{\prime} i N \widetilde{\Psi} B_{0} \\
& +p_{0}^{\prime} \mathcal{J}^{-1} \frac{\partial \widetilde{\psi}}{\partial \Theta}
\end{aligned}
$$

$\gamma \widetilde{\Psi} B_{0}=\mathcal{J}^{-1} \frac{\partial \widetilde{\phi}^{(2)}}{\partial \Theta}-i N \Lambda x \frac{V^{\prime} \mathcal{J}^{-1}}{4 \pi^{2}} \widetilde{\phi}+\eta \frac{\partial^{2}}{\partial \psi_{0}^{2}} \widetilde{\Psi} B_{0}\left|\vec{\nabla} \psi_{0}\right|^{2}$,

$$
\begin{aligned}
\gamma \rho \frac{\left|\vec{\nabla} \psi_{0}\right|^{2}}{B_{0}^{2}} \frac{\partial^{2} \widetilde{\phi}}{\partial \psi_{0}^{2}}-\left[\mathcal{J}^{-1} \frac{\partial}{\partial \Theta} \frac{\partial^{2}}{\partial \psi_{0}^{2}} \frac{\Psi^{(2)}\left|\vec{\nabla} \psi_{0}\right|^{2}}{B_{0}}\right. \\
\left.\quad-\frac{\partial \tilde{p}^{(2)}}{\partial \psi_{0}} \mathcal{J}^{-1} \frac{\partial}{\partial \Theta}\left(\frac{J_{\| 0}}{p_{0}^{\prime} B_{0}}\right)\right]+\frac{\partial \psi}{\partial \psi_{0}} \mathcal{J} \frac{\partial\left(J_{\| 0} / p_{0}^{\prime} B_{0}\right)}{\partial \Theta} \\
+i N \Lambda x \frac{V^{\prime} \mathcal{J}^{-1}}{4 \pi^{2}} \frac{\partial^{2}}{\partial \psi_{0}^{2}} \frac{\widetilde{\Psi} B_{0}\left|\vec{\nabla} \psi_{0}\right|^{2}}{B_{0}^{2}}-\tilde{p} \wedge\left|\vec{\nabla} \psi_{0}\right|^{2} 2 \kappa_{\psi} \\
+\frac{2 \tilde{p}}{B_{0}^{2}} \mathcal{J}^{-1} \frac{\partial}{\partial \Theta}\left(\frac{J_{\| 0}}{B_{0}}\right)=0,
\end{aligned}
$$

$$
\begin{aligned}
\frac{\gamma \tilde{p}}{\Gamma p_{0}}+ & {\left[\mathcal{J}^{-1} \frac{\partial}{\partial \Theta}\left(\frac{\widetilde{V}_{\|}^{(2)} B_{0}}{B_{0}^{2}}\right)-\frac{\partial \widetilde{\phi}^{(2)}}{\partial \psi_{0}} \mathcal{J}^{-1} \frac{\partial}{\partial \Theta}\left(\frac{J_{\| 0}}{p_{0}^{\prime} B_{0}}\right)\right] } \\
& -\eta \frac{\left|\vec{\nabla} \psi_{0}\right|^{2}}{B_{0}^{2}} \frac{\partial^{2} \tilde{p}}{\partial \psi_{0}^{2}}-i N \Lambda x \frac{V^{\prime} \mathcal{J}^{-1}}{4 \pi^{2}} \frac{\widetilde{V_{\|}} B_{0}}{B_{0}^{2}} \\
& +\widetilde{\phi}_{\wedge}\left|\vec{\nabla} \psi_{0}\right|^{2}\left(2 \kappa_{\psi}-\frac{p_{0}^{\prime}}{B_{0}^{2}}-\frac{p_{0}^{\prime}}{\Gamma p_{0}}\right)=0,
\end{aligned}
$$

where the superscript (2) denotes second-order variables in the expansion. 
Before considering the lowest order equations, note the second-order terms in Eqs. (C17) and (C18). To annihilate these problematic terms, the following operator is introduced:

$$
\langle Q\rangle=\frac{2 \pi}{V^{\prime}} \oint Q \mathcal{J} d \Theta
$$

which describes variables as functions of the resonant angle and flux surface label. If $Q$ is axisymmetric, this average is the same as a flux surface average. A useful relation for this average is

$$
\left\langle\mathcal{J}^{-1}\right\rangle=\frac{4 \pi^{2}}{V^{\prime}} .
$$

After annihilating the second-order terms in Eqs. (C17) and (C18), the equations will be in terms of $\left\langle\widetilde{V_{\|}} B_{0}\right\rangle$ and $\left\langle\widetilde{\Psi} B_{0}\right\rangle$, which we choose as our fundamental variables in addition to $\widetilde{\phi}$ and $\tilde{p}$.

We now consider the lowest-order equations. Equations (C13) and (C14) show $\tilde{p}=\langle\tilde{p}\rangle$ and $\tilde{\phi}=\langle\widetilde{\phi}\rangle$ are independent of $\Theta$ to lowest order. Equations (C15) and (C16) show that $\widetilde{V_{\|}} B_{0}$ and $\widetilde{\Psi} B_{0}$ have a $\Theta$ dependence in the lowest order. Solving for that dependence, we find

$\widetilde{V_{\|}} B_{0}=\left\langle\widetilde{V_{\|}} B_{0}\right\rangle \frac{B_{0}^{2}}{\left\langle B_{0}^{2}\right\rangle}+\frac{1}{p_{0}^{\prime}} \frac{\partial \widetilde{\phi}}{\partial \psi_{0}}\left(\frac{J_{\| 0}}{B_{0}} B^{2}-\left\langle\frac{J_{\| 0}}{B_{0}} B_{0}^{2}\right\rangle \frac{B_{0}^{2}}{\left\langle B_{0}^{2}\right\rangle}\right)$,

$$
\begin{aligned}
\frac{\partial^{2}}{\partial \psi_{0}^{2}} \widetilde{\Psi} B_{0}= & \frac{\partial^{2}}{\partial \psi_{0}^{2}}\left\langle\widetilde{\Psi} B_{0}\right\rangle \frac{\frac{B_{0}^{2}}{\left|\vec{\nabla} \psi_{0}\right|^{2}}}{\left\langle\frac{B_{0}^{2}}{\left|\vec{\nabla} \psi_{0}\right|^{2}}\right\rangle}+\frac{1}{p_{0}^{\prime}} \frac{\partial \tilde{p}}{\partial \psi_{0}} \\
& \times\left(\frac{\frac{J_{\| 0}}{B_{0}} B^{2}}{\left|\vec{\nabla} \psi_{0}\right|^{2}}-\left\langle\frac{J_{\| 0}}{B_{0}} \frac{B_{0}^{2}}{\left|\vec{\nabla} \psi_{0}\right|^{2}}\right\rangle \frac{\frac{B_{0}^{2}}{\left|\vec{\nabla} \psi_{0}\right|^{2}}}{\left|\frac{B_{0}^{2}}{\left|\vec{\nabla} \psi_{0}\right|^{2}}\right\rangle}\right) .
\end{aligned}
$$

We are now ready to consider the higher-order equations. The averaged parallel momentum equation is

$$
\gamma \rho\left\langle\widetilde{V_{\|}} B_{0}\right\rangle=i N \Lambda x \tilde{p}+p_{0}^{\prime} i N\left\langle\widetilde{\Psi} B_{0}\right\rangle .
$$

This equation contains no derivatives so it may be used to trivially eliminate $\left\langle\widetilde{V_{\|}} B_{0}\right\rangle$ from the other equations. The parallel Ohm's Law when averaged is

$$
\gamma\left\langle\widetilde{\Psi} B_{0}\right\rangle=-i N \Lambda x \widetilde{\phi}+\eta \frac{\partial^{2}}{\partial \psi_{0}^{2}}\left\langle\widetilde{\Psi} B_{0}\left|\vec{\nabla} \psi_{0}\right|^{2}\right\rangle
$$

Equation (C24) can be used to find an expression for the last term:

$$
\begin{aligned}
\frac{\partial^{2}}{\partial \psi_{0}^{2}}\left\langle\widetilde{\Psi} B_{0}\left|\vec{\nabla} \psi_{0}\right|^{2}\right\rangle & =\frac{\partial^{2}}{\partial \psi_{0}^{2}}\left\langle\widetilde{\Psi} B_{0}\right\rangle \frac{\left\langle B_{0}^{2}\right\rangle}{\left\langle B_{0}^{2} /\left|\vec{\nabla} \psi_{0}\right|^{2}\right\rangle}+\frac{1}{p_{0}^{\prime}} \frac{\partial \tilde{p}}{\partial \psi_{0}}\left(\left\langle\frac{J_{\| 0}}{B_{0}} B_{0}^{2}\right\rangle\right. \\
& \left.-\left\langle\frac{J_{\| 0}}{B_{0}} \frac{B_{0}^{2}}{\left|\vec{\nabla} \psi_{0}\right|^{2}}\right\rangle \frac{\left\langle B_{0}^{2}\right\rangle}{\left\langle B_{0}^{2} /\left|\vec{\nabla} \psi_{0}\right|^{2}\right\rangle}\right) .
\end{aligned}
$$

Using this equation, we rewrite Eq. (C26) as

$$
\frac{\partial^{2}}{\partial \psi_{0}^{2}}\left\langle\widetilde{\Psi} B_{0}\right\rangle=\frac{\gamma}{\eta C}\left\langle\widetilde{\Psi} B_{0}\right\rangle+\frac{i N \Lambda x}{\eta C} \widetilde{\phi}-\frac{\Lambda H}{p_{0}^{\prime}} \frac{\partial \tilde{p}}{\partial \psi_{0}},
$$

where

$$
\begin{aligned}
C= & \frac{\left\langle B_{0}^{2}\right\rangle}{\left\langle B_{0}^{2} /\left|\vec{\nabla} \psi_{0}\right|^{2}\right\rangle}, \\
H= & \frac{\left\langle B_{0}^{2} /\left|\vec{\nabla} \psi_{0}\right|^{2}\right\rangle}{\Lambda}\left(\frac{\left\langle\left(J_{\| 0} / B_{0}\right) B_{0}^{2}\right\rangle}{\left\langle B_{0}^{2}\right\rangle}\right. \\
& \left.-\frac{\left\langle\left(J_{\| 0} / B_{0}\right)\left(B_{0}^{2} /\left|\vec{\nabla} \psi_{0}\right|^{2}\right)\right\rangle}{\left\langle B_{0}^{2} /\left|\vec{\nabla} \psi_{0}\right|^{2}\right\rangle}\right) .
\end{aligned}
$$

The vorticity and pressure equations are more tedious to calculate because each have two second-order terms that must be annihilated. To obtain the annihilated vorticity equation, we use

$\langle$ Vorticity Equation〉

$$
\left.+\left\langle\frac{J_{\| 0}}{p_{0}^{\prime} B_{0}} \frac{\partial}{\partial \psi_{0}} \text { (Parallel Momentum Equation }\right)\right\rangle .
$$

Similarly, the annihilated pressure equation is formed using $\langle$ Pressure Equation〉

$$
\left.+\left\langle\frac{J_{\| 0}}{p_{0}^{\prime} B_{0}} \frac{\partial}{\partial \psi_{0}} \text { (Parallel Ohm's Law }\right)\right\rangle .
$$

When performing these averages, there are terms containing $\left(\partial / \partial \psi_{0}\right)\left\langle J_{\| 0} / B_{0} \widetilde{\Psi} B_{0}\right\rangle$. It is convenient to define a new variable to handle these terms:

$$
\begin{gathered}
\Gamma \equiv \frac{\left\langle B_{0}^{2} /\left|\vec{\nabla} \psi_{0}\right|^{2}\right\rangle}{\Lambda^{2}}\left[\frac{\left\langle\left(J_{\| 0} / B_{0}\right) B_{0}^{2}\right\rangle}{\left\langle B_{0}^{2}\right\rangle} \frac{\partial}{\partial \psi_{0}}\left\langle\widetilde{\Psi} B_{0}\right\rangle\right. \\
\left.-p_{0}^{\prime}\left\langle\frac{1}{B_{0}^{2}}\right\rangle \tilde{p}-\frac{\partial}{\partial \psi_{0}}\left\langle\frac{J_{\| 0}}{B_{0}} \widetilde{\Psi} B_{0}\right\rangle\right] .
\end{gathered}
$$

Using Eq. (C24), one can derive the following relation for $\Gamma$ :

$$
\frac{\partial \Gamma}{\partial \psi_{0}}=\frac{H}{\Lambda} \frac{\partial^{2}}{\partial \psi_{0}^{2}}\left\langle\widetilde{\Psi} B_{0}\right\rangle-\frac{F}{p_{0}^{\prime}} \frac{\partial \tilde{p}}{\partial \psi_{0}},
$$

where 


$$
\begin{aligned}
F= & \frac{\left\langle B_{0}^{2} /\left|\vec{\nabla} \psi_{0}\right|^{2}\right\rangle}{\Lambda^{2}}\left[p_{0}^{\prime 2}\left\langle\frac{1}{B_{0}^{2}}\right\rangle+\left\langle\frac{\left(J_{\| 0} / B_{0}\right)^{2} B_{0}^{2}}{\left|\vec{\nabla} \psi_{0}\right|^{2}}\right\rangle\right. \\
& \left.-\frac{\left.\left\langle\frac{J_{\|_{0}}}{B_{0}} \frac{B_{0}^{2}}{\left|\vec{\nabla} \psi_{0}\right|^{2}}\right\rangle^{2}\right]}{\left\langle\frac{B_{0}^{2}}{\left|\vec{\nabla} \psi_{0}\right|^{2}}\right\rangle}\right]
\end{aligned}
$$

To facilitate comparison with GGJ, we transform our variables to their scaled variables. The relations between their variables and ours are

$$
\begin{aligned}
& \Xi \equiv \frac{1}{\gamma} \tilde{\vec{V}} \cdot \vec{\nabla} \psi_{0}=-\frac{1}{\gamma} i N \tilde{\phi}, \\
& \bar{\Psi} \equiv-\frac{\left\langle\widetilde{\vec{B}} \cdot \vec{\nabla} \psi_{0}\right\rangle}{i N \Lambda L_{R}}=\frac{\left\langle\tilde{\Psi} B_{0}\right\rangle}{\Lambda L_{R}}, \\
& \Upsilon \equiv \frac{\widetilde{\vec{B}} \cdot \vec{B}_{0}}{p_{0}^{\prime}}=-\frac{\tilde{p}}{p_{0}^{\prime}},
\end{aligned}
$$

where

$$
L_{R}=\left[\frac{\mathcal{J}^{2} \rho \bar{M} \eta^{2}\left\langle B_{0}^{2}\right\rangle^{2}}{N^{2} q^{\prime 2}\left\langle B_{0}^{2} /\left|\vec{\nabla} \psi_{0}\right|^{2}\right\rangle^{2}}\right]^{1 / 6} .
$$

The growth rates and $x$ are also rescaled as

$$
\begin{aligned}
& Q=\frac{\gamma}{\left[\eta N^{2} q^{\prime 2}\left\langle B_{0}^{2}\right\rangle / \mathcal{J}^{2} \rho \bar{M}\left\langle B_{0}^{2} /\left|\vec{\nabla} \psi_{0}\right|^{2}\right\rangle\right]^{1 / 3}}=\frac{\gamma L_{R}^{2}}{\eta C}, \\
& X=\frac{x}{L_{R}} .
\end{aligned}
$$

With these variables our annihilated parallel Ohm's Law, annihilated vorticity, annilated pressure, and gamma equation become

$$
\begin{aligned}
& \bar{\Psi}_{X X}-H \Upsilon_{X}=Q(\bar{\Psi}-X \Xi), \\
& Q^{2} \Xi_{X X}-Q X^{2} \Xi+E \Upsilon+Q X \Psi+\Gamma=0, \\
& Q \Upsilon_{X X}-X^{2} \Upsilon-Q^{2} G \Upsilon+(G-K E-R) Q^{2} \Xi+X \bar{\Psi} \\
& \quad-K Q^{2} \Gamma=0, \\
& \Gamma_{X}=H \bar{\Psi}_{X X}+F \Upsilon_{X},
\end{aligned}
$$

where

$$
\begin{aligned}
& \Lambda=\frac{4 \pi^{2} q^{\prime}}{V^{\prime}}, \quad C=\frac{\left\langle B_{0}^{2}\right\rangle}{\left\langle B_{0}^{2} /\left|\vec{\nabla} \psi_{0}\right|^{2}\right\rangle}, \\
& G=\frac{\left\langle B_{0}^{2}\right\rangle}{\Gamma p_{0} \bar{M}}, \quad K=\frac{\Lambda^{2} C}{p_{0}^{\prime 2} \bar{M}}, \quad R=\frac{1}{\bar{M}}\left\langle B_{0}^{2}\right\rangle\left\langle\frac{1}{B_{0}^{2}}\right\rangle,
\end{aligned}
$$

$$
\begin{aligned}
& H=\frac{\left\langle B_{0}^{2} /\left|\vec{\nabla} \psi_{0}\right|^{2}\right\rangle}{\Lambda}\left(\frac{\left\langle\left(J_{\|_{0}}\right) B_{0}^{2}\right\rangle}{\left\langle B_{0}^{2}\right\rangle}\right. \\
& \left.-\frac{\left\langle\left(J_{\|_{0}} / B_{0}\right)\left(B_{0}^{2} /\left|\vec{\nabla} \psi_{0}\right|^{2}\right)\right\rangle}{\left\langle B_{0}^{2} /\left|\vec{\nabla} \psi_{0}\right|^{2}\right\rangle}\right], \\
& E=\frac{\left\langle\frac{B_{0}^{2}}{\left|\vec{\nabla}_{0}\right|^{2}}\right\rangle}{\Lambda^{2}}\left[2 p_{0}^{\prime}\left\langle\kappa_{\psi}\right\rangle-p_{0}^{\prime 2}\left\langle\frac{1}{B_{0}^{2}}\right\rangle\right] \text {, } \\
& F=\frac{\left\langle B_{0}^{2} /\left|\vec{\nabla} \psi_{0}\right|^{2}\right\rangle}{\Lambda^{2}}\left[p_{0}^{\prime 2}\left\langle\frac{1}{B_{0}^{2}}\right\rangle+\left\langle\frac{\left\langle J_{\| 0} / B_{0}\right)^{2} B_{0}^{2}}{\left|\vec{\nabla} \psi_{0}\right|^{2}}\right\rangle\right. \\
& \left.-\frac{\left\langle\left(J_{\|_{0}} / B_{0}\right)\left(B_{0}^{2} /\left|\vec{\nabla} \psi_{0}\right|^{2}\right)\right\rangle^{2}}{\left\langle B_{0}^{2} /\left|\vec{\nabla} \psi_{0}\right|^{2}\right\rangle}\right] \text {, } \\
& \bar{M}=\left\langle\frac{B_{0}^{2}}{\left|\vec{\nabla} \psi_{0}\right|^{2}}\right\rangle\left[\left\langle\frac{\left|\vec{\nabla} \psi_{0}\right|^{2}}{B_{0}^{2}}\right\rangle+\frac{1}{p_{0}^{\prime 2}}\left(\left\langle\left(\frac{J_{\|_{0}}}{B_{0}}\right)^{2} B_{0}^{2}\right\rangle\right.\right. \\
& \left.\left.-\frac{\left\langle\frac{J_{\|_{0}}}{B_{0}} B_{0}^{2}\right\rangle^{2}}{\left\langle B_{0}^{2}\right\rangle}\right)\right]
\end{aligned}
$$

and $N$ is the toroidal mode number.

${ }^{1}$ H. R. Strauss, Phys. Fluids 19, 134 (1976).

${ }^{2}$ H. R. Strauss, Phys. Fluids 20, 1354 (1977).

${ }^{3}$ J. F. Drake and T. M. Antonsen, Phys. Fluids 27, 898 (1984).

${ }^{4}$ R. Izzo, D. A. Monticello, J. DeLucia, W. Park, and C. M. Ryu, Phys. Fluids 28, 903 (1985).

${ }^{5}$ R. D. Hazeltine and J. D. Meiss, Phys. Rep. 121, 1 (1985); R. D. Hazeltine and J. D. Meiss, Plasma Confinement (Addison-Wesley, Redwood City, CA, 1992).

${ }^{6}$ H. R. Strauss, J. Plasma Phys. 57, 83 (1997).

${ }^{7}$ J. D. Callen, W. X. Qu, K. D. Siebert, B. A. Carreras, K. C. Shaing, and D. A. Spong, in Plasma Physics and Controlled Nuclear Fusion Research 1986, Kyoto (International Atomic Energy Agency, Vienna, 1987), Vol. 2, p. 157.

${ }^{8}$ J. E. Menard, S. C. Jardin, C. E. Kessel, and J. Manickam, Nucl. Fusion 37, 595 (1997).

${ }^{9}$ R. L. Miller, Y. R. Lin-Liu, A. D. Turnbull, V. S. Chan, L. D. Pearlstein, O. Sauter, and L. Villard, Phys. Plasmas 4, 1062 (1997).

${ }^{10}$ R. L. Dewar, J. Plasma Fusion Res. 73, 1123 (1997).

${ }^{11}$ B. Coppi, J. M. Greene, and J. L. Johnson, Phys. Fluids 6, 101 (1966).

${ }^{12}$ J. C. Strikwerda, Finite Difference Schemes (Chapman and Hall, New York, 1989).

${ }^{13}$ J. P. Freidberg, Ideal Magnetohydrodynamics (Plenum, New York, 1987).

${ }^{14}$ E. Hameiri, Phys. Fluids 26, 230 (1983).

${ }^{15}$ F. L. Hinton and R. D. Hazeltine, Rev. Mod. Phys. 48, 239 (1976).

${ }^{16}$ S. P. Hirshman and D. J. Sigmar, Nucl. Fusion 9, 1079 (1981).

${ }^{17}$ T. A. Gianakon, C. C. Hegna, and J. D. Callen, Phys. Plasmas 5, 4637 (1996).

${ }^{18}$ J. L. Johnson and J. M. Greene, Plasma Phys. 9, 611 (1967).

${ }^{19}$ A. H. Glasser, J. M. Greene, and J. L. Johnson, Phys. Fluids 18, 875 (1975).

${ }^{20}$ J. M. Greene, in Introduction to Resistive Instabilities (Ecole Polytechnique Federale de Lausanne, Lausanne, Switzerland, 1976). 